\title{
Un lion des cavernes (Panthera (Leo) spelaea) exploité au Dryas récent : les données du gisement du Peyrat (Saint-Rabier, Dordogne, France)
}

Philippe Fosse, Stéphane Madelaine, Christine Oberlin, Catherine Cretin et Peggy Bonnet-Jacquement

\section{CpenEdition}

\section{Journals}

Édition électronique

URL : http://journals.openedition.org/paleo/3098

DOI : $10.4000 /$ paleo.3098

ISSN : 2101-0420

Éditeur

SAMRA

Édition imprimée

Date de publication : 30 décembre 2017

Pagination : 91-115

ISSN : 1145-3370

Référence électronique

Philippe Fosse, Stéphane Madelaine, Christine Oberlin, Catherine Cretin et Peggy Bonnet-Jacquement, «Un lion des cavernes (Panthera (Leo) spelaea) exploité au Dryas récent : les données du gisement du Peyrat (Saint-Rabier, Dordogne, France) », PALEO [En ligne], 28 | 2017, mis en ligne le 01 juin 2018, consulté le 07 juillet 2020. URL : http://journals.openedition.org/paleo/3098 ; DOI : https://doi.org/ 10.4000/paleo.3098

\section{cc) (†) $\ominus$}

PALEO est mis à disposition selon les termes de la licence Creative Commons Attribution - Pas d'Utilisation Commerciale - Pas de Modification 4.0 International. 


\title{
Un lion des cavernes (Panthera (Leo) spelaea) exploité au Dryas récent : les données du gisement du Peyrat (Saint-Rabier, Dordogne, France)
}

\author{
Philippe FOSSE ${ }^{(a)}$, Stéphane MADELAINE(b), Christine OBERLIN(c), \\ Catherine CRETIN(b) ${ }^{(b)}$ Peggy BONNET-JACQUEMENT ${ }^{(b)}$
}

\begin{abstract}
Résumé : Dans les années 1960, les fouilles du gisement du Peyrat (Saint-Rabier, Dordogne) par A. Cheynier ont livré un abondant matériel lithique et osseux, rapporté au Magdalénien et à l'Azilien. La stratigraphie complexe, décrite à partir de cinq tranchées profondes, comprend une dizaine de couches d'inégale puissance dans lesquelles de très nombreux vestiges paléontologiques ont été recueillis. Ponctuellement déterminée (carnets de terrain de Cheynier) à partir de restes dentaires, la faune se compose principalement du renne et du cerf. La révision de quelques pièces paléontologiques déterminables a permis à l'un des auteurs (SM) d'identifier la présence du lion des cavernes à partir d'une colonne vertébrale sub-complète (sept vertèbres cervicales, six vertèbres thoraciques, six vertèbres lombaires), rapportée à un individu mâle. La présence de ce Félidé dans un contexte stratigraphique de l'extrême fin du Tardiglaciaire, dans un horizon culturel azilien récent, est étayée par une date radiométrique directe de $10590 \pm 70 \mathrm{BP}$ (SacA-38311), soit -10755/-10455 cal BC $(2 \sigma)$. Cette date est, à ce jour, la plus récente jamais obtenue pour cette espèce à l'échelle eurasiatique. De plus, deux vertèbres (atlas, $12^{e}$ vertèbre thoracique) présentent des traces de découpe. Le présent article dresse un bilan géo-chronologique des restes du lion des cavernes dans le Sud-Ouest de la France (nord Aquitaine - versant sud des Pyrénées) et souligne l'intérêt des gisements régionaux dans les études taphonomiques et zooarchéologiques concernant la disparition de la grande faune glaciaire.
\end{abstract}

Mots-clés : lion des cavernes, Panthera (Leo) spelaea, Tardiglaciaire, Dryas récent, radiocarbone, ostéométrie, traces de découpe, Dordogne.

Abstract: A Human exploitation of a cave lion (Panthera (Leo) spelaea) in the Younger Dryas: the data from Le Peyrat site (Saint-Rabier, Dordogne, France). In the 1960s, excavations of the archaeological site of Le Peyrat (Saint-Rabier, Dordogne) by A. Cheynier delivered an important lithic and bone sample, related to the Magdalenian and to the Azilian cultures. A complex stratigraphy, mainly described from 5 deep trenches, includes about ten layers of uneven thickness yielding numerous paleontological remains. The macrofauna was summary identified (Cheynier's field notes) and mainly consists of reindeer and red deer remains. A first revision of faunal remains by one of the Authors (SM) allows to identify a subcomplete vertebral column (i.e. 7 cervical vertebrae, 6 thoracic vertebrae and 6 lumbar vertebrae) belonging to a cave lion male. The presence of this large Felid in a recent Late Glacial stratigraphical level (Late Azilian), is supported by a direct radiocarbon date of $10590 \pm 70 \mathrm{BP}$ (SacA-38311), or 10755/-10455 cal BC (2 $\sigma)$. So far, this date is the most recent ever obtained for this species in Eurasia. Furthermore, cutmarks were found on two vertebrae (atlas and 12th thoracic vertebra). The present article is a survey of the geo-chronological distribution of the cave lion in Southwestern France (from North Aquitaine up to the Spanish Pyrenees) and underlines the importance of regional sites in taphonomic and zooarchaeological studies dealing with the extinction of the large cold fauna during Late Glacial.

Key-words: cave lion, Panthera (Leo) spelaea, Late Glacial, Younger Dryas, radiocarbon date, bone measurement, cutmarks, Dordogne.

(a) Aix Marseille Univ, CNRS, Minist Culture \& Com, LAMPEA, MMSH, UMR 7269, 5 rue du Château de l'Horloge, BP 647, 13094 Aix-en-Provence, France, cedex 2 - fosse@mmsh.univ-aix.fr

(b) Musée National de Préhistoire, 1 rue du Musée, FR-24620 Les Eyzies de Tayac \& Université Bordeaux, UMR 5199 (PACEA), bâtiment B8, allée Geoffroy Saint-Hilaire, CS 50023, FR-33615 Pessac cedex - stephane.madelaine@culture.gouv.fr ; catherine.cretin@culture.gouv.fr ; peggy.jacquement@culture.gouv.fr

(c) Université Lyon 1, UMR 5138 CNRS, Bât. Carbone 14, 40 boulevard Niels Bohr, Campus de La Doua, FR-69622 Villeurbanne christine.oberlin@univ-lyon1.fr 


\section{Introduction}

À partir du Pléistocène moyen, les lions l.s. sont régulièrement mentionnés dans les cortèges fauniques d'Europe (Dietrich 1968 ; Schütt 1969 ; Bonifay 1971 ; Clot 1980 ; Sala 1990 ; Argant 1991 ; Barycka 2008 ; Marciszak \& Sefaniak 2010 ...). Suite aux révisions de diverses séries paléontologiques d'Eurasie (Ballésio 1980 ; Sotnikova \& Nikolskiy 2006 ; Barycka 2008 ; Marciszak \& Stefaniak 2010 ; Hemmer 2011; Sotnikova \& Foronova 2014) et aux analyses paléogénétiques (Barnett et al. 2009, 2016), le lion des cavernes du Pléistocène supérieur (Panthera (Leo) spelaea Goldfuss, 1810) est décrit comme une espèce distincte du lion d'Afrique (Panthera (Leo) leo Linné, 1758), avec une séparation au niveau du sous-genre. Cette forme pourrait ne pas provenir du très grand lion de la première moitié du Pléistocène moyen (Panthera (Leo) fossilis Reichenau, 1906).

Fréquent, comme les autres grands Carnivores (ours des cavernes, hyène des cavernes), dans les séries archéologiques et paléontologiques depuis le stade isotopique 7 (MIS 7) jusqu'à la première moitié du stade isotopique 3 (MIS 3), le lion des cavernes perdure, seul semble-t-il, à partir du stade isotopique 2 (MIS 2) avant de disparaître au cours du stade isotopique 1 (MIS 1) (Stuart \& Lister 2011).

Dans le Sud-Ouest de la France, les deux versants pyrénéens et la cordillère cantabrique, le lion des cavernes $a$, depuis le XIXème siècle, été signalé dans une " quarantaine de gisements " côté français (Clot \& Duranthon $1990: 86$ ) et dans une quinzaine de cavités côté espagnol (Altuna 1972 ; Arribas 2004). Cette vaste région géographique présente l'originalité d'avoir livré des squelettes complets de lion des cavernes d'une part (Filhol \& Filhol 1871 ; Boule 1906 ; Altuna 1972, 1981 ; Costamagno et al. 1997 ; Castaños 2005) et des restes osseux de cette espèce dans des niveaux tardifs du Paléolithique supérieur d'autre part, sous forme de vestiges paléontologiques bruts (La Tourasse (Harlé 1893), Urtiaga (Altuna 1972), Le Morin (Delpech 1967, 1975, 1983)) ou travaillés (dents percées à Duruthy (Lartet \& Chaplain Duparc 1874), Les Espélugues, Les Scilles (Saint Périer, 1926)). Ces mentions, supposant une survivance locale de l'espèce très tardive, n'ont retrouvé leur réelle importance qu'avec la récente découverte de restes de lion des cavernes présentant des traces de découpe dans l'Azilien du Closeau dans le bassin parisien et datés de $12248 \pm 66$ uncal BP (Bodu \& Mevel 2008), soit $-12598 /-12003$ cal BC $(2 \sigma)$. Cette date est considérée comme l'une des plus récentes car un important travail de datage direct de restes de lion des cavernes pour l'Eurasie et l'Amérique du Nord (110 gisements) suggère globalement la disparition de cette espèce aux alentours de 15000 ans (cal BP) en Eurasie et plus tardivement, vers 11 000-12 000 ans (cal BP) en Amérique du Nord (Stuart \& Lister, op. cit.). L'identification d'éléments squelettiques, découpés, d'un lion des cavernes en Dordogne, dans un contexte du «Magdalénien » et daté directement, permet de préciser la chronologie de la disparition de cette espèce et d'aborder les relations groupes humains - lion des cavernes au cours du Paléolithique d'un point de vue économique et symbolique.

\section{Présentation du gisement et révision chrono-culturelle préliminaire}

Le gisement du Peyrat est localisé sur la commune de SaintRabier en Dordogne. Une barre rocheuse, globalement orientée Sud - Nord et accessible par un imposant talus, a été prospectée par Cheynier entre 1958 et 1967 (CleyetMerle 1988). Cinq tranchées perpendiculaires au pied de falaise ont été réalisées dans des dépôts de pente. Quatre d'entre elles ont livré des informations stratigraphiques, du Sud au Nord : les tranchées C, N, N', élargie en N" et NN. L'extrémité nord de trois d'entre elles (N, N'/N" et NN) se termine en abris ou grottes en pied de falaise (la " grotte supérieure » pour N, la " plate-forme du four » pour N'/N" et la « grotte inférieure » pour NN) et leur stratigraphie est difficilement corrélable à celle des tranchées, leur dynamique de remplissage étant probablement différente. Les notes manuscrites de Cheynier, courageusement synthétisées par J.-J. Cleyet-Merle à partir de données restreintes (absence des descriptions du Mésolithique), font état d'un remplissage conséquent dans le talus (plus de sept mètres de sédiments composent "la » stratigraphie (tranchée $\mathrm{N}$ ) considérablement bouleversée (solifluxion, bioturbations locales dues à des garennes)). Un niveau de référence, la " barrière de tuf " sépare nettement deux ensembles culturels, un inférieur (couches $H, G, F, E, D 3$, $\mathrm{D} 2$ et $\mathrm{D} 1$ du talus), qui pourrait être rattaché à une phase ancienne du Magdalénien et un Magdalénien supérieur Azilien (couches C2, C1, B, A du talus) mais ne se retrouve pas dans les locus en pied de falaise. Les stratigraphies à l'intérieur des grottes (C3NV, C2NV, C1NV, BNV et ANV) sont donc difficiles à corréler avec celles du dépôt de pente même si elles font a priori partie de l'ensemble supérieur.

Les décomptes (in Cleyet-Merle, op. cit) font état de 140042 produits de débitage et 5463 outils. En l'absence d'équivalence certaine entre les niveaux archéologiques perçus dans les tranchées $\mathrm{N}, \mathrm{N}^{\prime} / \mathrm{N}^{\prime \prime}$ et $\mathrm{NN}$ et les remplissages en grotte, J.-J. Cleyet-Merle a proposé une synthèse archéologique du site, une étude typologique et l'identification des pièces diagnostiques. Par exemple, plusieurs harpons ont été signalés dans l'ensemble supérieur, dont un fragment de harpon plat (Cleyet-Merle 1988 : 344, fig. $8 n^{\circ}$ 9). Parmi les pièces remarquables, notons le fragment d'os gravé sur ses deux faces de deux animaux (Cheynier, 1964a), une antilope saïga et un second animal indéterminé sur une face, un cheval et une croupe sur la deuxième ( $\gamma 2$ de la tranchée $\mathrm{N}$ ', correspondant à $\mathrm{C} 2$ de la tranchée $\mathrm{N})^{1}$. La colonne vertébrale de lion des cavernes, qui fait l'objet du présent

(1) Cette pièce a été improprement décrite comme baguette demi-ronde en ivoire (Cheynier 1964a, p. 25 ; Cleyet-Merle 1988, p. 343). 
article, a été trouvée dans la " grotte supérieure », dans la couche C2NV. Cheynier avait proposé une attribution de ce niveau au Magdalénien final, au même titre que la couche sus-jacente B/BNV. La couche ANV était, elle, proposée comme azilienne (Cheynier 1964b). Or, l'évolution des connaissances sur cette période du Tardiglaciaire et un rapide examen des séries lithiques et osseuses conservées au Musée national de Préhistoire (la collection étant un dépôt du Musée d'Archéologie nationale) nous amènent (PBJ, CC) à reconsidérer l'attribution chrono-culturelle des unités stratigraphiques de l'ensemble supérieur, et en particulier de la couche C2NV, probablement azilienne. La présence d'un harpon azilien, d'un fragment percé et biseauté de bois de cerf interprété comme un sifflet par Cheynier et d'un poinçon est contradictoire avec celle d'un harpon à double rang de barbelure et décor en partie basilaire (fig. 1). De même, l'industrie lithique présente des caractères aziliens, avec une majorité de grattoirs courts, souvent corticaux, sur éclat ou éclat laminaire, ainsi que des pointes aziliennes (monopointes à dos courbe) (fig. 2). La présence de deux pointes à base rétrécie, caractéristiques d'un Azilien récent, est à signaler. L'attribution de la couche C2NV à l'Azilien est d'autant plus plausible que l'on remarque la présence de nombreux rectangles et pointes de Malaurie dans la couche susjacente (CNV ou r1V). C'est alors la présence du harpon magdalénien (Cleyet-Merle 1988, n4, fig.8, p.343) dans cette même couche, manifestement laborienne, qui pose problème. Une révision complète des séries lithiques ainsi qu'une reconsidération de la dynamique des remplissages, si elle est possible, s'avèrent nécessaires. Enfin, il faut noter la présence de différents squelettes humains, dont au moins un (LP5) est synchrone de l'Azilien car directement daté de $11430 \pm 150$ BP (Samsel et al. 2016 : 213).

À ce jour, aucune certitude autre que celle d'un gisement datant de l'extrême fin du Paléolithique supérieur n'est disponible. En dépit de ces lacunes indéniables, l'identification du lion des cavernes dans le gisement du Peyrat présente un intérêt certain car l'espèce n'a jamais été identifiée dans un contexte archéologique aussi récent.

\section{Matériel et méthode}

Les vestiges paléontologiques prévus d'être confiés à $\mathrm{J}$. Bouchud n'ont, semble-t-il, pas été étudiés. Par conséquent, l'essentiel des informations provient des carnets de fouilles rédigés quotidiennement par Cheynier. Dans ces notes concernant les niveaux CNV et C2NV, la découverte progressive de « grosses vertèbres, parfois en connexion " est consignée, entre le 6 juin et le 14 août 1961. Au moins 14 vertèbres sont mentionnées et correspondent probablement à la colonne vertébrale présentée dans cet article. Dans son compte rendu final de 1961, Cheynier cite les espèces qu'il a identifiées dans ces niveaux : " lapin (abondant), renne, cerf, mégacéros, sanglier, bison, cheval, capridés, loup, chat sauvage, rongeurs, petits carnassiers, microfaune rare, oiseaux, poissons, castor ». À la suite de cette liste, « le rachis presque complet d'un gros animal » est rapporté, avec réserve, au bison même si sur l'atlas était écrit " cerf ". Aujourd'hui, la colonne vertébrale comprend 19 pièces, formant deux portions distinctes (fig. 3). Le premier tronçon comprend les sept vertèbres cervicales (C1-C7) associées aux trois premières vertèbres thoraciques (Th1-Th3) et sont toutes marquées C2NV. La seconde portion regroupe les trois dernières vertèbres thoraciques (Th11-Th13) en connexion avec les six vertèbres lombaires ; la onzième vertèbre thoracique et les quatre premières vertèbres lombaires n'ont pas d'attribution de couche alors que les deux dernières lombaires sont attribuées à C2NV, la douzième vertèbre thoracique est rapportée à la fois à CNV et C2NV et la treizième thoracique à « VC2 ».

La révision de ces restes paléontologiques a été engagée par l'un des présents auteurs (SM) en vue d'être présentés au public visitant le Musée National de Préhistoire. Bien qu'articulées solidement entre elles entravant leur libre manipulation et souvent dégradées au niveau de leurs apophyses transverses, les vertèbres, notamment l'atlas et l'axis, présentent des caractéristiques morphologiques et ostéométriques propres aux Félidés et particulièrement au lion des cavernes. Rarement utilisées à des fins d'identification principale, les vertèbres constituent dans le cas présent l'unique source de documentation paléontologique, une recherche d'autres éléments postcéphaliques ou dentaires de grands Carnivores parmi plusieurs dizaines de portoirs s'étant révélée infructueuse. Les descriptions anatomiques reposent sur la nomenclature éditée par Barone (1976) et les mesures relevées sur les codes de mesurage prescrits par von den Driesch (1976). Pour ces dernières, afin de disposer d'un corpus homogène et suffisant, des correspondances entre différentes publications ont été nécessaires aussi bien pour l'atlas (fig. 4) que l'axis (fig. 5). Les données comparatives rassemblées concernent le léopard (Panthera pardus Linné, 1758) moderne (Krofel 2004) et pléistocène (Altuna 1972 ; Sanchiz et al. 2015), le lion moderne (exemplaires mesurés au Musée National de Préhistoire (collection L. Pales), Museum d'Histoire naturelle de Toulouse et Musée Requien à Avignon ; Gross 1992 ; Krofel, ibid), le lion des cavernes (Baume Longue, Lherm, Soulabé (cette étude), Romain la Roche (Argant 2010), Herdengel (Pacher, 2009)). Pour cette espèce, certains échantillons ont parfois été sexés, à partir des restes dentaires lorsque le squelette est (sub)complet (individus mâles de Montmaurin Coupe Gorge (cette étude), Arrikrutz, Lezetxiki, VI (Altuna, 1981) et Siegsdorf (Gross 1992)), individus femelles d'Azoleta (Castaños, 2005), Potocka zijalka (Krofel, ibid)). Enfin des données ostéométriques concernant le lion d'Amérique (Panthera atrox Leidy, 1853) ont été incorporées à cette étude à partir des incomparables séries de Rancho la Brea (Merriam \& Stock, 1932). Pour les dates radiocarbone, les âges ont été calibrés à l'aide de la courbe IntCal13 (Reimer et al., 2013) et du logiciel OxCal 4.3 (Bronk Ramsey 2009). Les résultats sont présentés en cal BP et en cal BC. 


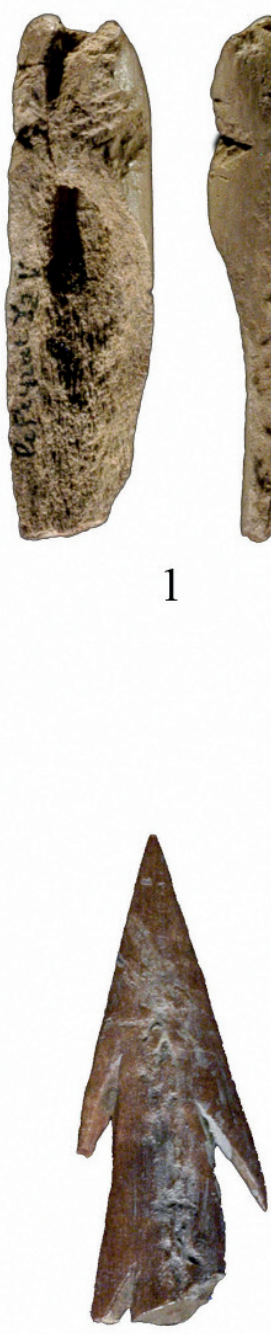

2

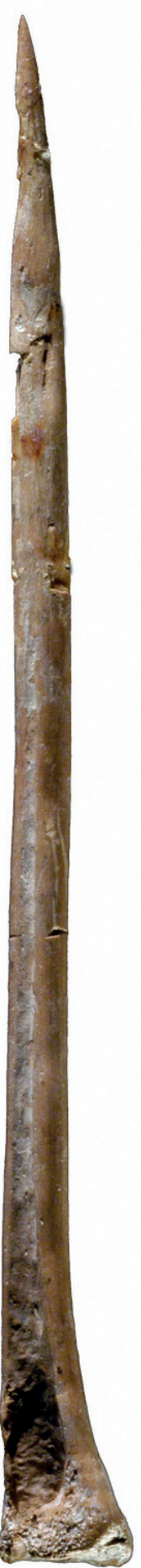

3

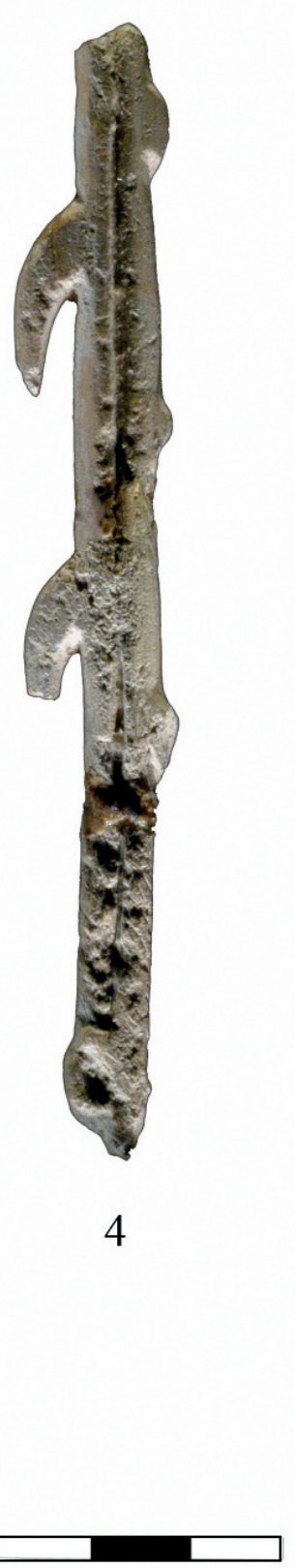

Figure 1 - Industrie osseuse du Peyrat provenant de la " grotte supérieure " et rattachée à l'ensemble C2NV. 1 : fragment percé et biseauté de bois de cerf (" sifflet ») ; 2 : harpon azilien ; 3 : poinçon ; 4 : harpon à double rang de barbelure décoré (photos Ph. Jugie, MNP). Les pièces 1 et 2 sont marquées r2V, ce qui équivaut semble-t-il à C2NV. Les pièces 3 et 4 sont malheureusement non marquées. Toutes figurent comme appartenant à C2NV dans l'article de J.-J. CleyetMerle (1988, $n^{\circ}$ 1, 2, 5 et 9 , fig. 8, p. 343 ; les autres pièces de cette figure ont été écartées car elles portent un marquage indiquant une provenance légèrement différente).

Figure 1 - Bone industry from Le Peyrat, coming from the "upper cave " and assigned to level C2NV. 1: perforated and beveled fragment of deer antler (" whistle »); 2: azilian harpoon head; 3 : punch; 4 : decorated harpoon head with double range of barbs (photos Ph. Jugie, MNP). Numbers 1 and 2 are marqued $\mathrm{r} 2 \mathrm{~V}$, equivalent to C2NV. Numbers 3 and 4 are unfortunately unmarqued. All of them are belonging to C2NV in the paper of J.-J. Cleyet-Merle (1988, $n^{\circ}$ 1, 2, 5 and 9, fig. 8, $p$. 343; the other pieces have been unselected because of their marking indicating a quite different origin).

\section{Résultats}

Chez les Félidés actuels, la colonne vertébrale est composée d'une cinquantaine de vertèbres, réparties comme suit : sept cervicales, 13 thoraciques, sept lombaires, trois sacrées et 20-24 caudales (Barone 1976). La restitution des deux portions vertébrales du Peyrat et le rang anatomique des vertèbres reposent sur l'homologie des zones de contacts entre, d'une part, $\mathrm{C} 7$ et la première vertèbre présentant une surface articulaire pour les côtes 

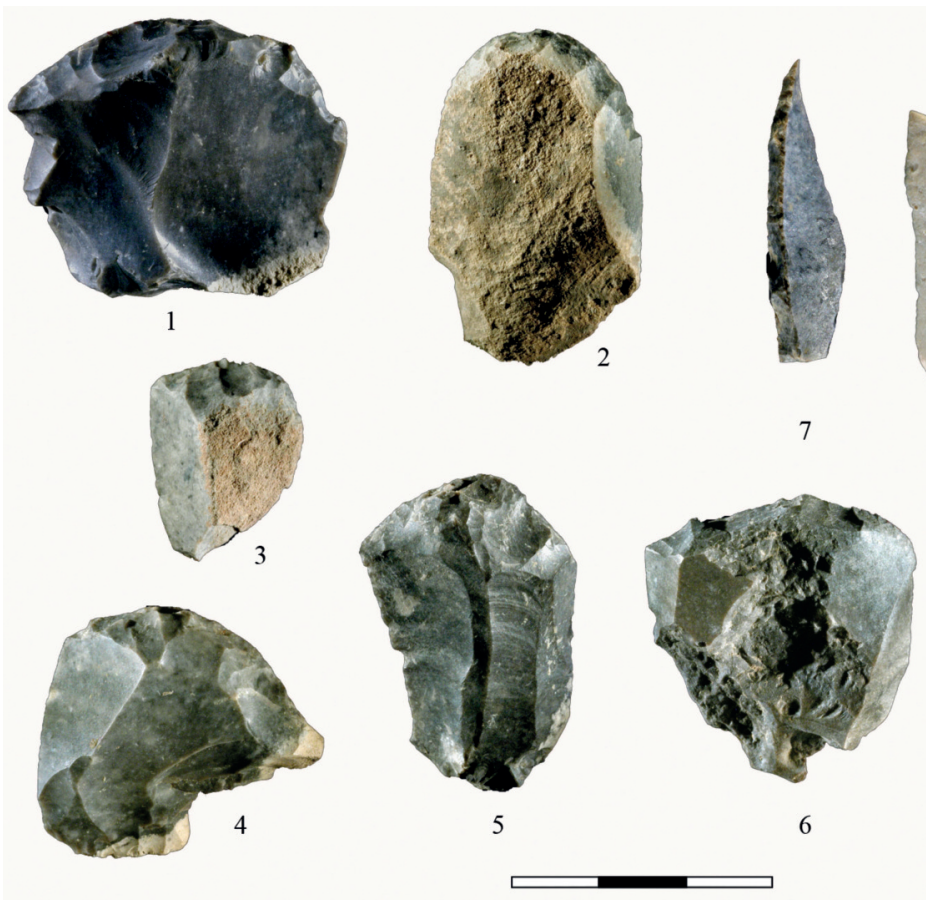

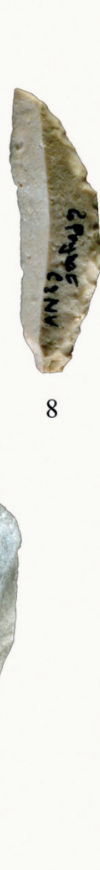

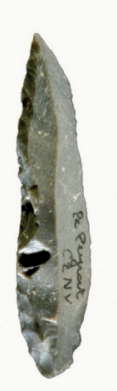

9

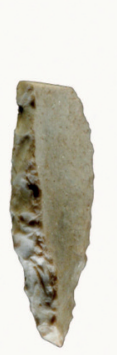

11

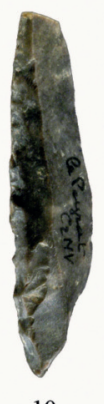

10

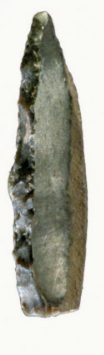

12
Figure 2 - Industrie lithique du Peyrat provenant de la " grotte supérieure » et rattachée à l'ensemble C2NV. 1 à 6 : grattoirs aziliens ; 7 à 12 : pointes aziliennes (monopointes à dos courbe et pointes à dos rectiligne); les $n^{o s} 10$ et 11 sont à base rétrécie (photos Ph. Jugie, MNP). Les pièces retenues pour cette planche sont toutes marquées C2NV.

Figure 2 - Lithic industry from Le Peyrat, coming from the " upper cave " and assigned to level C2NV. 1 to 6: azilian endscrapers; 7 to 12: azilian points (single backed point, both curved and straight); numbers 10 and 11 have a shrinked base (photos Ph. Jugie, MNP). Tools in this plate are all marqued C2NV.
(Th1) et d'autre part entre la dernière vertèbre de morphologie semblable (soit Th13) et six vertèbres avec des processus transverses nettement développés et dépourvues d'articulation pour les côtes (soit les 6 premières lombaires, L1-L6). L'attribution au lion des cavernes repose sur les dimensions ainsi que sur la morphologie des différentes faces des deux premières vertèbres cervicales.

\section{L'atlas}

L'atlas du Peyrat est fragmentaire (fig. 6A), les ailes sont cassées, tout comme une partie crâniale de l'arc dorsal. Le corps est néanmoins préservé et le faible développement des tubercules dorsal et ventral, proéminents chez les Ongulés (Artiodactyles), le dégagement très net de la partie articulaire crâniale par rapport aux ailes (forte incisure plongeant jusqu'au niveau des foramens intervertébraux), l'épaisseur de la pièce étant haute et non comprimée, décrivant une ouverture vertébrale plus ronde qu'ovale ne permettent pas d'attribuer cet atlas ni à un cerf ni à un autre Ongulé mais à un Carnivore. Comme chez le lion moderne (et les autres grands félins), l'incisure crâniale est décrochée " en paliers » depuis les extrémités latérales jusqu'au niveau du foramen vertébral, tandis qu'elle est régulière, droite transversalement à l'axe de la pièce chez les ours (bruns). L'arc dorsal, en vue caudale, présente une courbure régulière et peu marquée chez le lion moderne ou fossile, une courbure un peu plus accentuée chez l'ours brun et une courbure très forte (en $U$ inversé) chez l'ours des cavernes. Le diamètre dorso-ventral de l'arc dorsal est épais chez Ursus spelaeus (développement du tubercule dorsal parfois marqué) alors que qu'il est mince chez tous les autres Carnivores (lion, ours brun, hyène des cavernes).
II est à noter que chez Crocuta spelaea, l'épaisseur de l'arc dorsal est identique à celle de l'arc ventral.

Les mesures relevées sur l'atlas du Peyrat (tabl. 1) sont systématiquement supérieures aux exemplaires modernes et pléistocènes attribués au léopard et s'accordent davantage aux données recueillies sur les lions pléistocènes d'Europe et d'Amérique. Un sexage peut être proposé (cf. infra).

\section{L'axis}

La deuxième vertèbre cervicale du Peyrat est relativement bien préservée (fig. 6B), les destructions majeures se situant au niveau du processus épineux des parties terminales des processus transverses. La dent est particulièrement longue, se dégageant nettement du corps de la vertèbre, caractéristique des Carnivores et non des Ongulés ; le processus épineux forme, en vue caudale, un triangle avec un sommet nettement plus effilé que chez l'ours brun (arrondi) ; de même, les foramens intervertébraux sont, en vue latérale, nettement plus bas, plus étirés antéro-postérieurement et plus loin de la surface articulaire crâniale que chez les Ursidae (U. arctos et $U$. spelaeus). Enfin, en vue ventrale, l'angle de l'écartement processus transverse - corps de la vertèbre est plus fermé chez les félins que chez les ours. L'exemplaire du Peyrat présente toutes ces caractéristiques, propres aux Félidés.

Les différentes mesures relevées sur l'axis du Peyrat (tabl. 2) témoignent d'une pièce aux dimensions nettement supérieures aux axis de léopards pléistocènes, s'insérant dans la variabilité notée dans le groupe des lions d'Europe et d'Amérique. Sensiblement plus petit que les deux échantillons de Romain la Roche, l'axis du Peyrat est en 


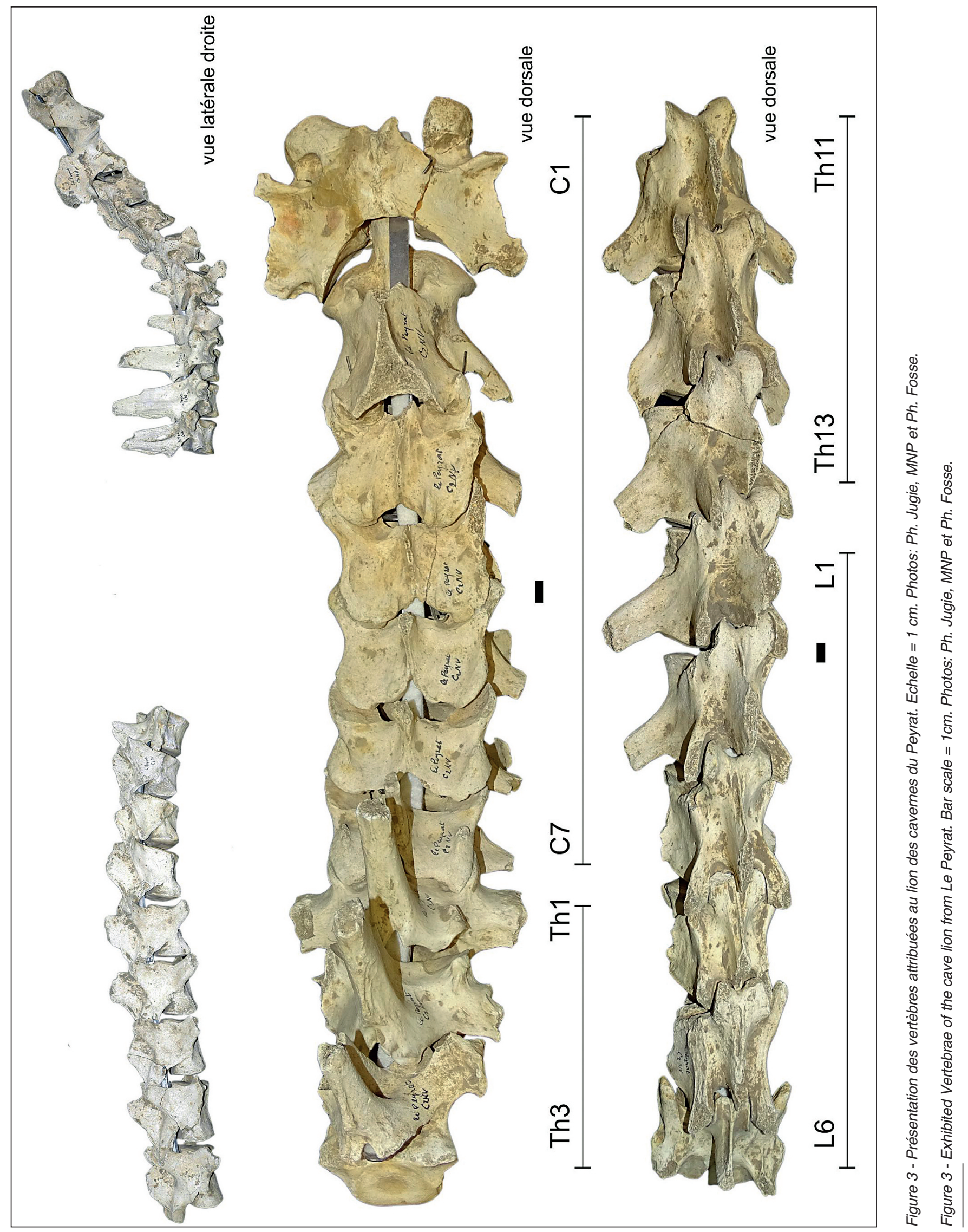



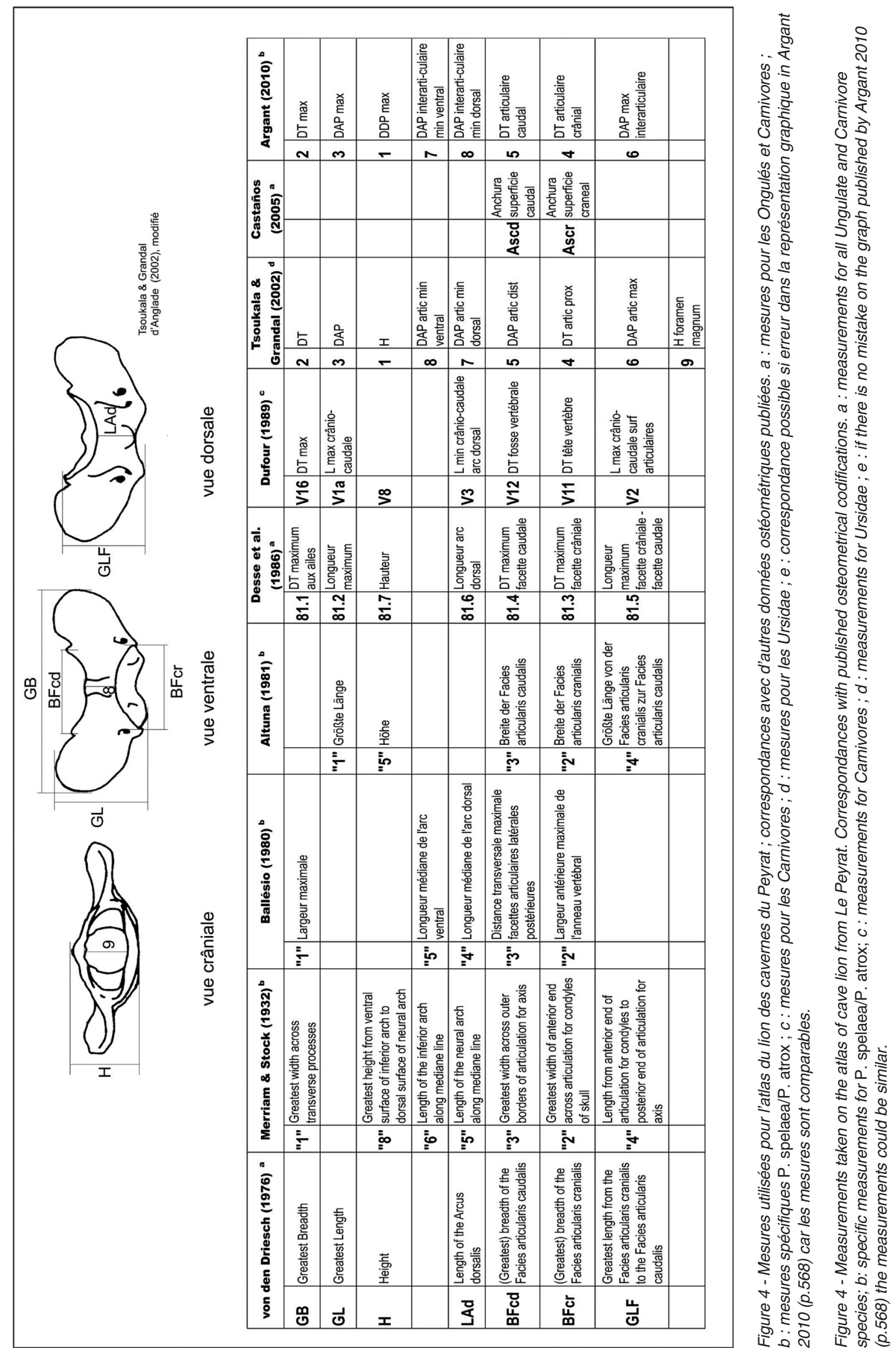


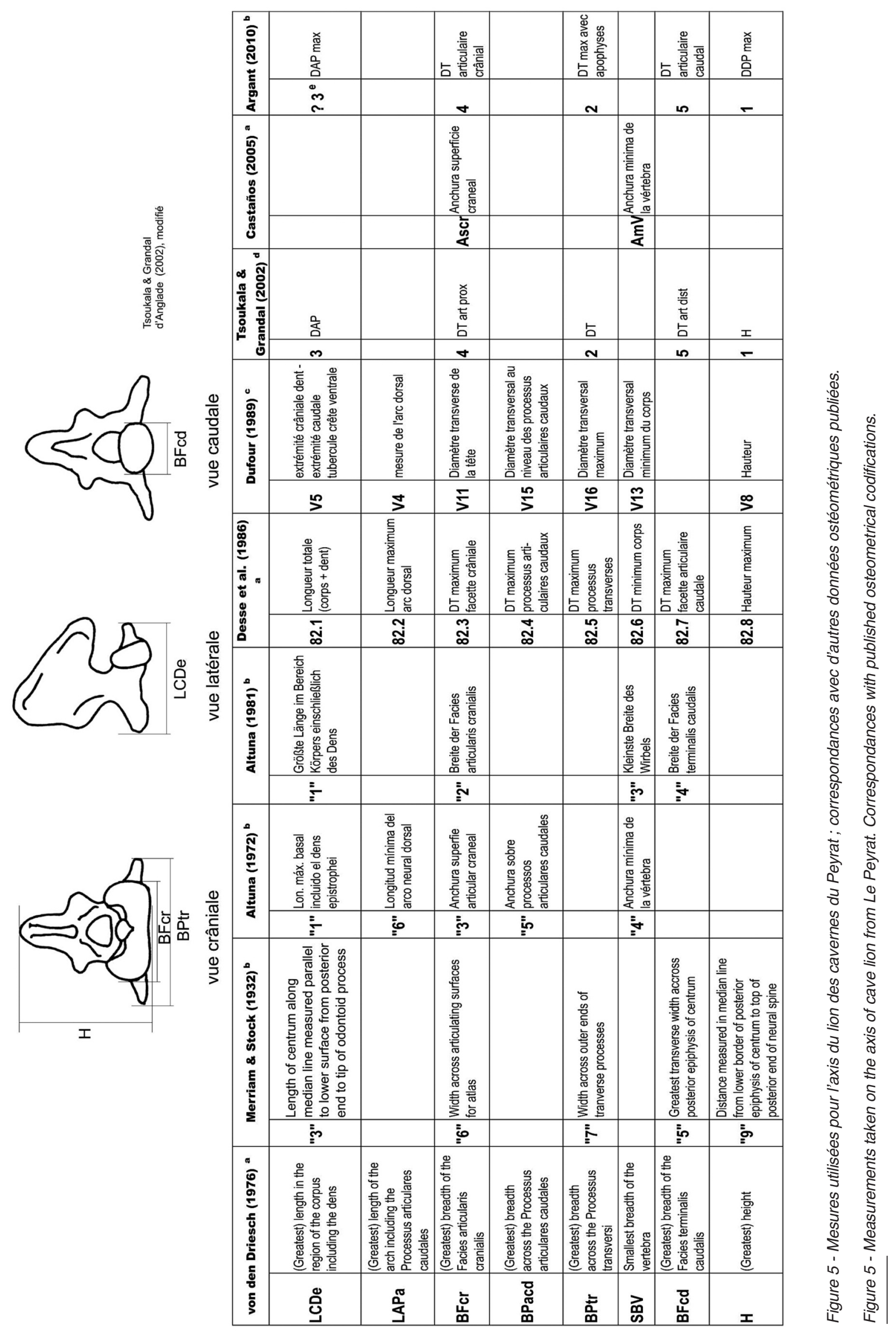




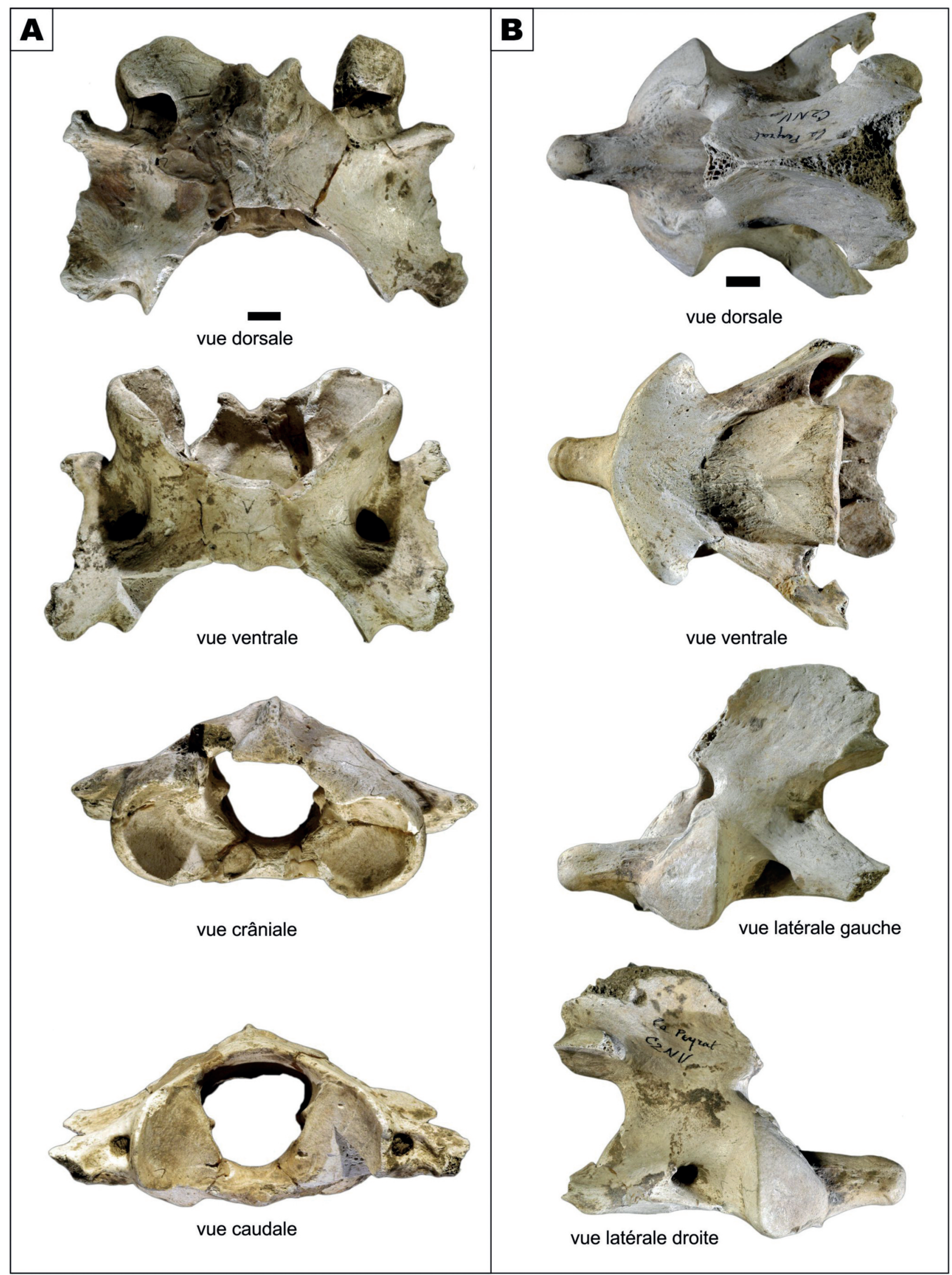

Figure 6 - Atlas et axis du lion des cavernes du Peyrat. Echelle $=1 \mathrm{~cm}$. Photos : Ph. Jugie, MNP.

Figure 6 - Atlas and axis of the cave lion from Le Peyrat. Bar scale $=1 \mathrm{~cm}$. Photos: Ph. Jugie, MNP. 


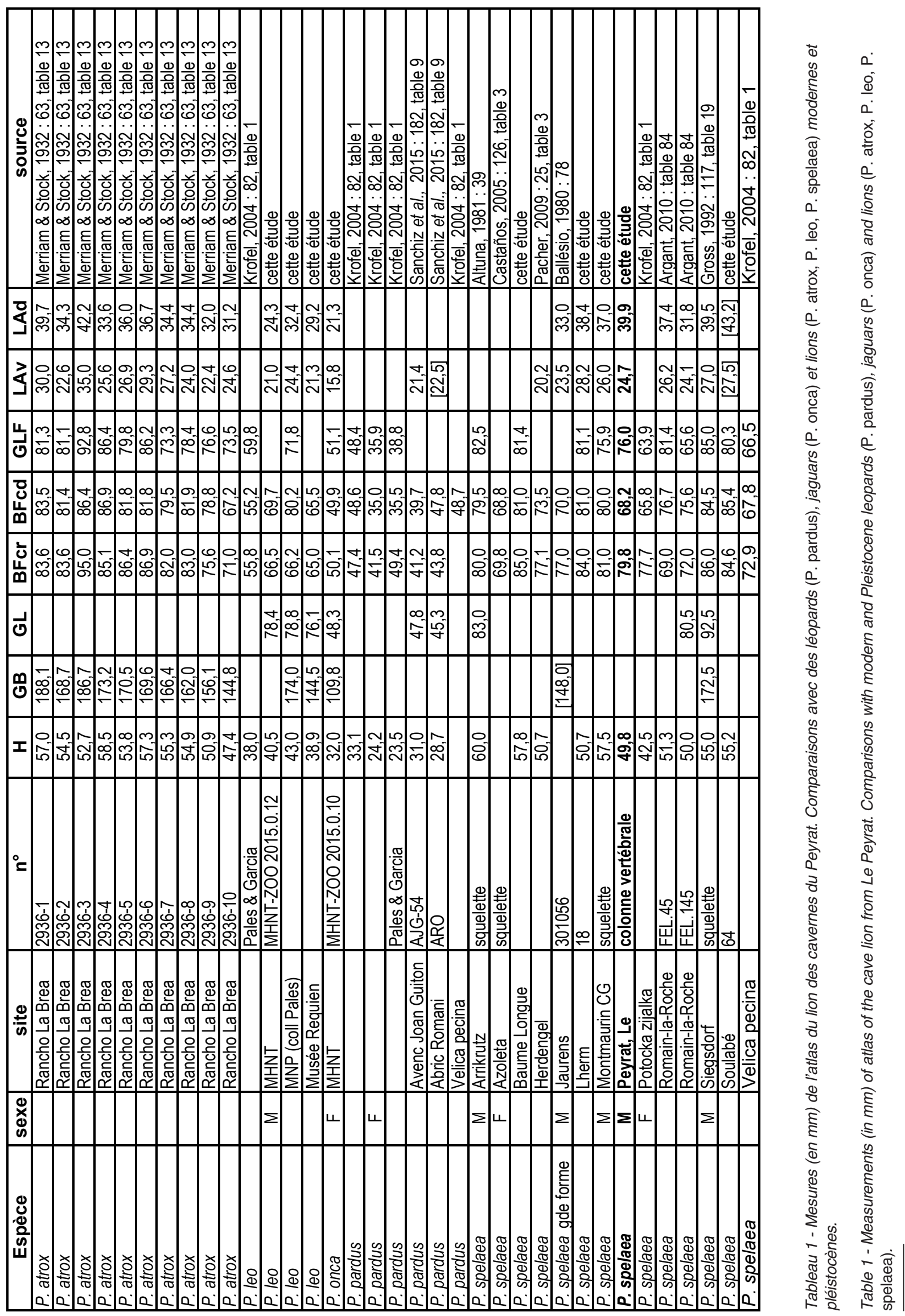




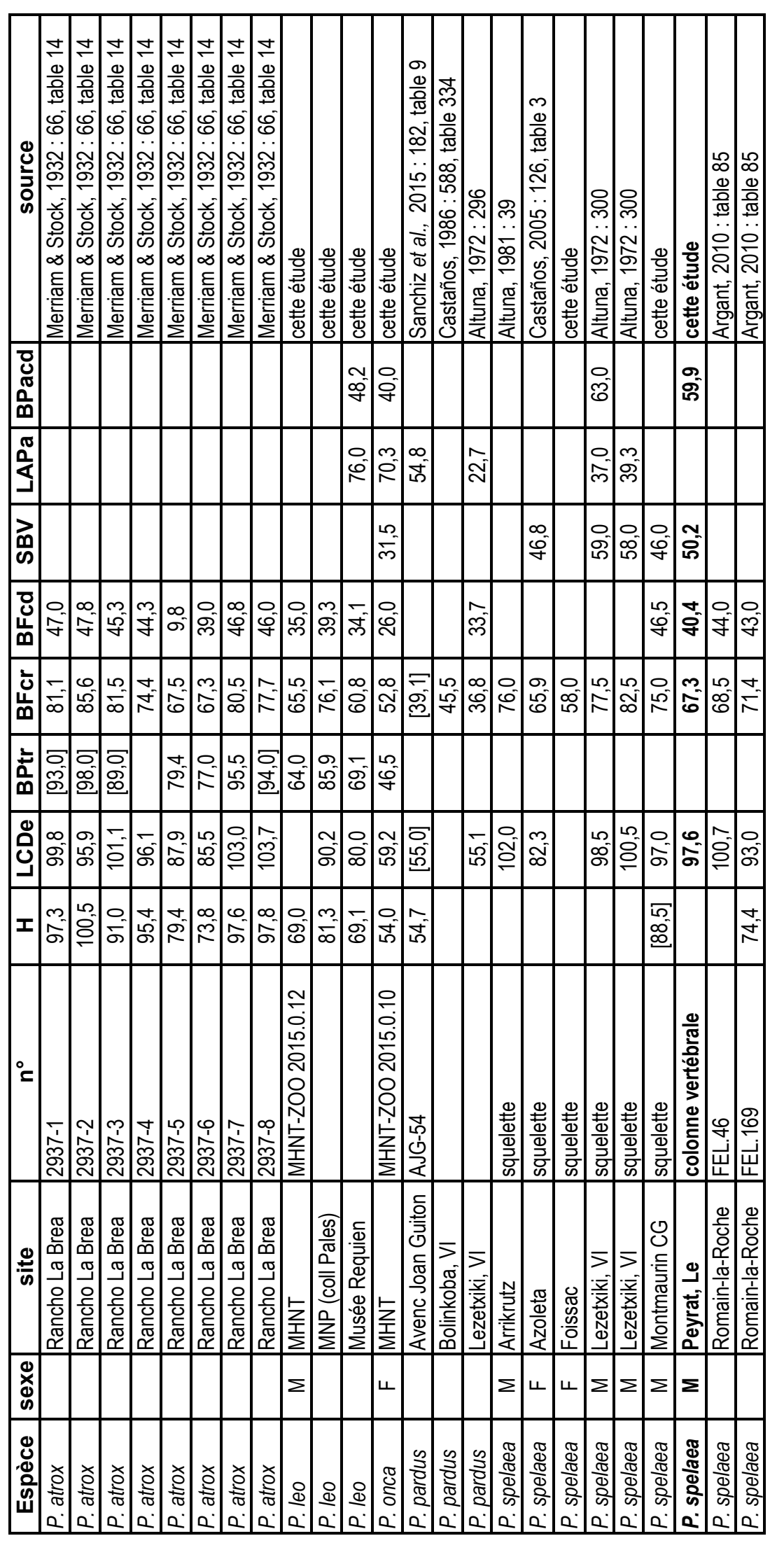

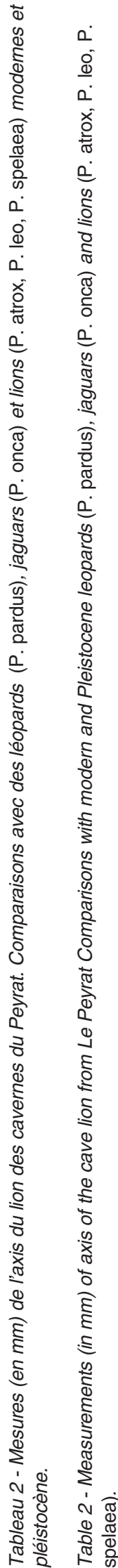


deçà des mesures publiées pour les mâles des grands lions du Pléistocène moyen final / Pléistocène supérieur initial mais se démarque des individus femelles de comparaison. Comme pour l'atlas, un sexage est avancé (cf. infra).

\section{Sexage de la colonne vertébrale du Peyrat}

Chez les Félidés modernes, le dimorphisme sexuel est important (10-20 \% entre mâles et femelles) et presque tous les éléments du squelette (dents isolées, os longs, métapodes, carpiens, tarsiens) permettent une détermination sexuelle, à partir des données uni- ou bivariées (Turner 1984 ; Gross 1992 ; Meachen Samuels \& Binder 2009). Appliquées au site de Jaurens, daté du MIS 3 et qui a livré une série abondante de lion (NRD $=240$; $\mathrm{NMI}=6$ d'après Ballésio 1980), les deux formes (petite et grande), initialement décrites comme distinctes (Ballésio, op. cit., suivant les remarques de Clouet (1891) et Boule (1906)) ont été ré-attribuées respectivement aux femelles et mâles (Turner, op. cit.). Cette considération, largement acceptée depuis (Kurten,1985 ; Diedrich \& Rathgeber 2012), est importante car le site de Jaurens a livré un atlas de la grande forme de lion des cavernes, partiellement mesurable, pouvant donc être sexé (mâle). De même, le squelette sub-complet recueilli dans la grotte d'Azoleta en Navarre a finalement été assigné à une femelle eu égard aux petites dimensions des restes dentaires et postcéphaliques (Castaños 2005).

La longueur totale des sept vertèbres cervicales du Peyrat affiche un peu plus de $357 \mathrm{~mm}$; sur l'individu mâle, relativement grêle, de Cajarc (non sexé par Boule (1906) mais dont les mesures sur tous les éléments squelettiques postcrâniens et crâniens s'intègrent dans la variabilité des individus mâles recensés pour toute l'Europe (Fosse, inédit)), cette mesure est de $310 \mathrm{~mm}$ (Boule 1906 : 12).

L'atlas du Peyrat présente des mesures sensiblement équivalentes à la "grande forme» de Jaurens (Ballésio 1980), sans jamais atteindre cependant les dimensions relevées sur des individus robustes de la fin du Pléistocène moyen et de la première moitié du Pléistocène supérieur (fig. 7A). Les valeurs sont en revanche supérieures aux quelques données mentionnées pour les lionnes d'Azoleta (Castaños 2005) et de Potocka zijalka (Krofel 2004). L'atlas est attribué à un sujet mâle, de taille comparable à un lion moderne.

L'axis, bien que moins documenté que l'atlas d'un point de vue ostéométrique (fig. 7B), s'avère être plus robuste que la pièce mesurée pour la femelle d'Azoleta (Castaños 2005) et sensiblement plus petit que les mâles des grandes formes (ante MIS 3) d'Arrikrutz, Lezetxiki ou Montmaurin. Cette pièce peut d'autant plus être rapprochée d'un sujet mâle qu'une mesure (BFcr) prise in situ sur l'axis du squelette de Foissac a donné $58 \mathrm{~mm}$, suggérant une femelle (Fosse \& Madelaine, obs. pers.). La deuxième vertèbre cervicale du Peyrat peut donc être rapportée à un sujet mâle.

\section{La datation radiocarbone}

Retrouvés dans un contexte chrono-culturel particulièrement récent du Tardiglaciaire, les restes attribués au lion des cavernes ont fait l'objet d'un prélèvement pour une analyse radiocarbone afin de préciser la contemporanéité de cette espèce avec les autres taxons d'une part et de disposer d'une première date directe pour le lion des cavernes dans le Sud-Ouest d'autre part.

L'analyse effectuée par le laboratoire de Lyon sur un fragment prélevé sur l'atlas a fourni une date AMS de 10590 +/- 70 BP non calibrée (SacA-38311), soit -10755/10455 cal BC (2 $\sigma)$; il s'agit de la quatrième date radiocarbone directe obtenue sur os de lion des cavernes en France (tabl. 3). Ce résultat confirme à la fois la survivance du lion des cavernes jusqu'à l'extrême fin du Tardiglaciaire en Europe de l'Ouest, en rajeunissant d'environ 1500 ans sa présence dans la région, et l'appartenance de cette colonne vertébrale à une séquence archéologique, initialement attribuée au Magdalénien final mais manifestement plus récente, comme l'attestent à la fois l'examen de l'industrie et le résultat radiométrique. II est donc possible d'envisager l'extinction de l'espèce à partir de la seconde moitié du Dryas récent.

\section{Traces d'activité humaine ... et animale}

Sur la face ventrale de la douzième vertèbre thoracique figuraient à l'encre de Chine un repère pour signaler la présence d'incisions rectilignes et profondes. L'examen de toutes les pièces composant cette portion de colonne vertébrale confirme ces observations anciennes et a permis d'en reconnaître de nouvelles, sur l'atlas (fig. 8).

Sur la première vertèbre cervicale, des stries sont visibles sur les faces dorsale et ventrale. Sur la face dorsale, une incision isolée, particulièrement profonde, débute à quelques millimètres du tubercule dorsal pour rejoindre obliquement le bord médial du foramen intervertébral gauche. Une seconde strie, de même profondeur quoique plus courte, part du bord latéral de ce foramen et glisse jusqu'au rebord médial de l'aile. Toujours dans cette zone de l'atlas, au-dessus de la première strie décrite, trois fines entailles (une sub-parallèle et deux obliques) longent le bord de l'arc dorsal. Sur cette même face dorsale, dans le prolongement du tubercule dorsal, vers l'arc ventral, deux stries courtes et obliques sont visibles. Une troisième zone présente des traces de découpe, faisant miroir aux premières observations : vers le foramen intervertébral droit, trois incisions (deux entrecroisées, une isolée) ont marqué la surface corticale et une quatrième, profonde, parcourt le rebord médial de l'aile. Enfin, dans une dernière zone, vers l'arc ventral, apparaît une très longue et fine strie parallèle à la courbure de l'arc. Sur la face ventrale, des incisions répétées entaillent le bord de la cavité articulaire du condyle occipital et une strie isolée incise obliquement l'épaisseur de l'arc dorsal. Enfin en vue latérale gauche, une plage importante de très longues et fines stries sur les rebords externes dorsal et ventral de la cavité articulaire des condyles. La disposition de toutes ces marques 


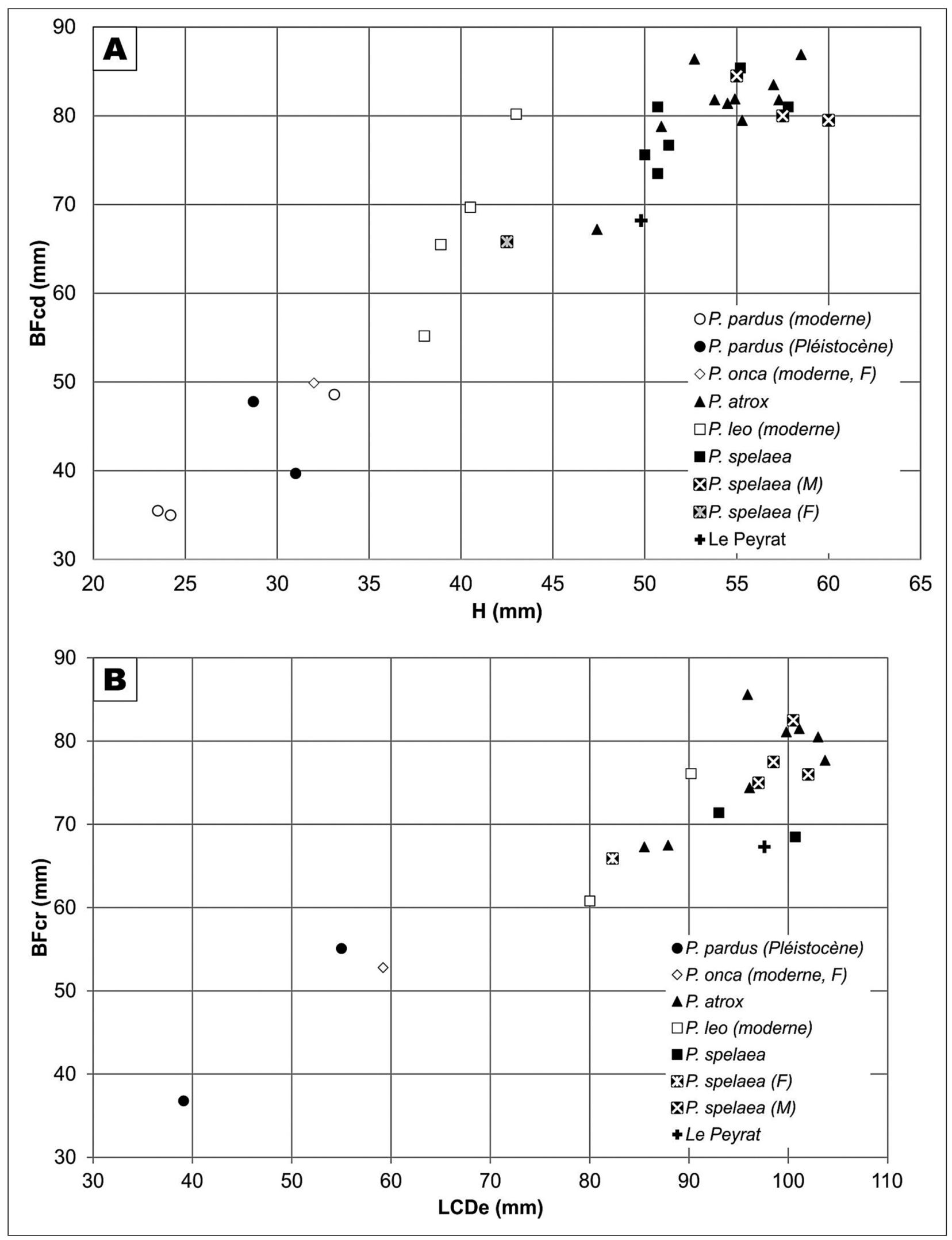

Figure 7 - Données ostéométriques comparées de I 'atlas $(A)$ et I 'axis (B) du lion des cavernes du Peyrat avec d'autres grands félins modernes et pléistocènes. Source : Altuna (1981), Argant (2010), Gross (1992), Krofel (2004), Merriam \& Stock (1932), Pacher (2009), Sanchiz et al. (2015).

Figure 7 - Comparative osteometrical data for atlas $(A)$ and axis of the cave lion from Le Peyrat with other large modern and pleistocene felids. source : Altuna (1981), Argant (2010), Gross (1992), Krofel (2004), Merriam \& Stock (1932), Pacher (2009), Sanchiz et al. (2015). 


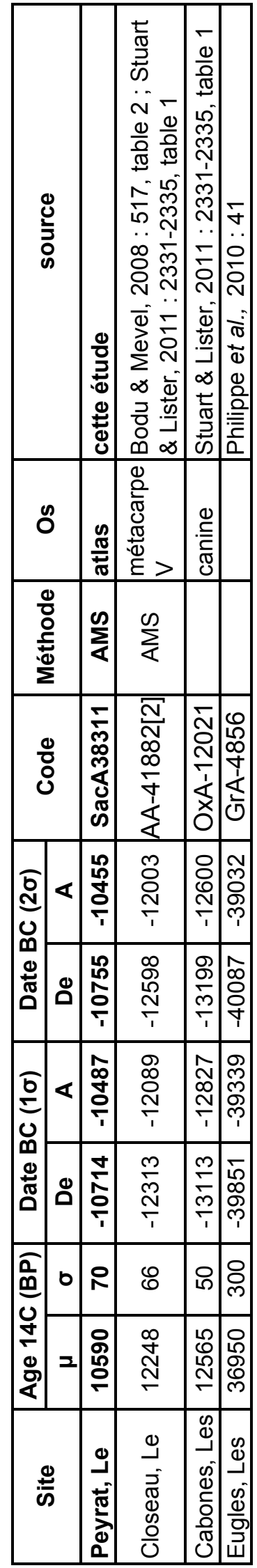

correspond partiellement à la typologie de Binford (1981 : 111,137 ) relative à la désarticulation (séparation tête tronc).

Sur la douzième vertèbre thoracique, partiellement endommagée dans sa partie latérale droite, six incisions particulièrement profondes et nettes, perpendiculaires à l'axe de l'os, parcourent la face ventrale du corps vertébral. D'autres marques, plus fines et superficielles, sont également visibles dans cette zone et vers le rebord du corps vertébral caudal. Sur le processus articulaire caudal, plusieurs stries obliques apparaissent à la surface de l'os. Toutes ces marques peuvent être rapportées à une activité d'éviscération et de désarticulation. En complément de ces traces anthropiques, au moins trois perforations (punctures) produites par des Carnivores sont identifiables sur cette pièce ; si la perforation isolée jouxte une strie de découpe, les deux enfoncements contigus recouvrent en revanche une incision et s'avèrent postérieurs à l'exploitation anthropique. L'attribution spécifique de ces traces de dents, qui ne dépassent pas quatre millimètres dans leur plus grande longueur, pourrait être recherchée dans les petits Canidés.

\section{Discussion}

Le lion des cavernes est fréquent dans les gisements du Sud-Ouest, depuis la deuxième moitié du Pléistocène moyen (11 sites) et tout au long du Pléistocène supérieur (184 gisements recensés dont 81 attribuables aux différentes chrono-cultures du Paléolithique supérieur, cf. Annexe). La plupart des gisements ne renferme que quelques restes appartenant à un seul individu, dont le statut taphonomique (occupant du site, proie, gibier) se pose.

Après avoir été particulièrement abondant durant les stades MIS 5,4 et 3 , en contexte de tanières (hyène et ours des cavernes) et anthropiques (Moustérien et surtout Aurignacien), le lion des cavernes perdure dans le Sud de l'Europe, jusqu'à l'extrême fin du Tardiglaciaire. A la revue de quelques gisements français, espagnols et italiens dans lesquels l'espèce a été identifiée entre 20000 et 10000 ans, la grotte du Peyrat constitue un des repères les plus fiables parmi les évidences de la persistance du lion des cavernes au Tardiglaciaire.

\section{Le lion des cavernes entre 20000 et 10000 ans BP : les datations directes}

En Eurasie, une vingtaine de gisements renfermant du lion des cavernes a livré des dates radiocarbone directes, comprises entre 20000 et 10000 ans (fig. 9). Si la plupart des dates proviennent du Nord Est eurasiatique, en contexte naturel, les résultats, pour les sites de l'Europe de l'Ouest sont intéressants car issus de séries stratifiées.

En Russie (Oural), plusieurs sites (Viasher 2, Verkhnegubakhinskaya, Podsemnich Ochotnikov : Stuart \& Lister 2011) attestent de la présence du lion des cavernes jusque -14000 ans cal BC. Dans le Nord de la Sibérie, l'espèce perdure un peu plus tardivement, jusqu'à - 


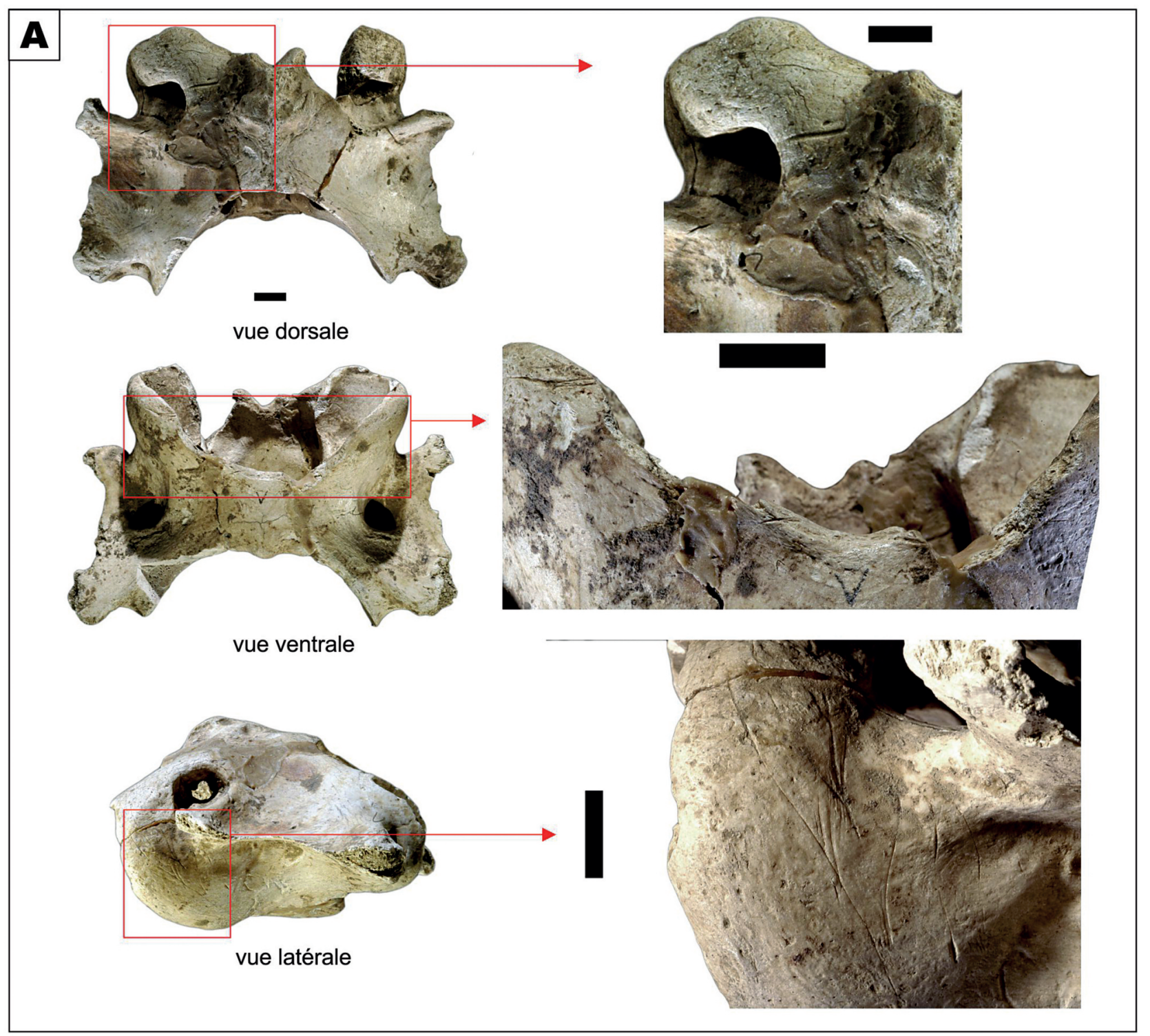

\section{$\mathbf{B}$}
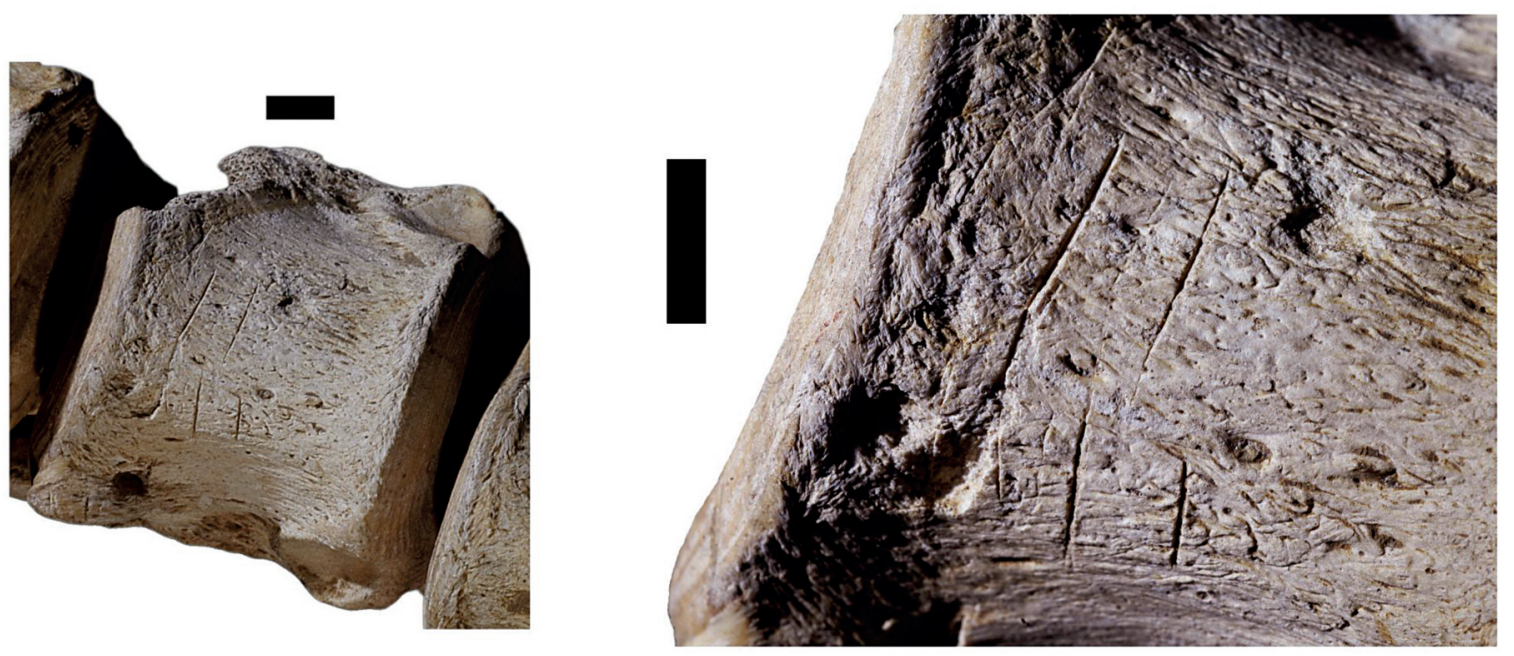

Figure 8 - Traces d'activité humaine et animale relevées sur I 'atlas $(A)$ et la vertèbre thoracique $(B)$ du lion des cavernes du Peyrat. échelle $=1 \mathrm{~cm}$. photos Ph. Jugie, MNP.

Figure 8 - Human and Carnivore traces on the atlas $(A)$ and the thoracic vertebra $(B)$ of the cave lion from Le Peyrat. bar scale $=1 \mathrm{~cm}$. photos Ph. Jugie, MNP. 


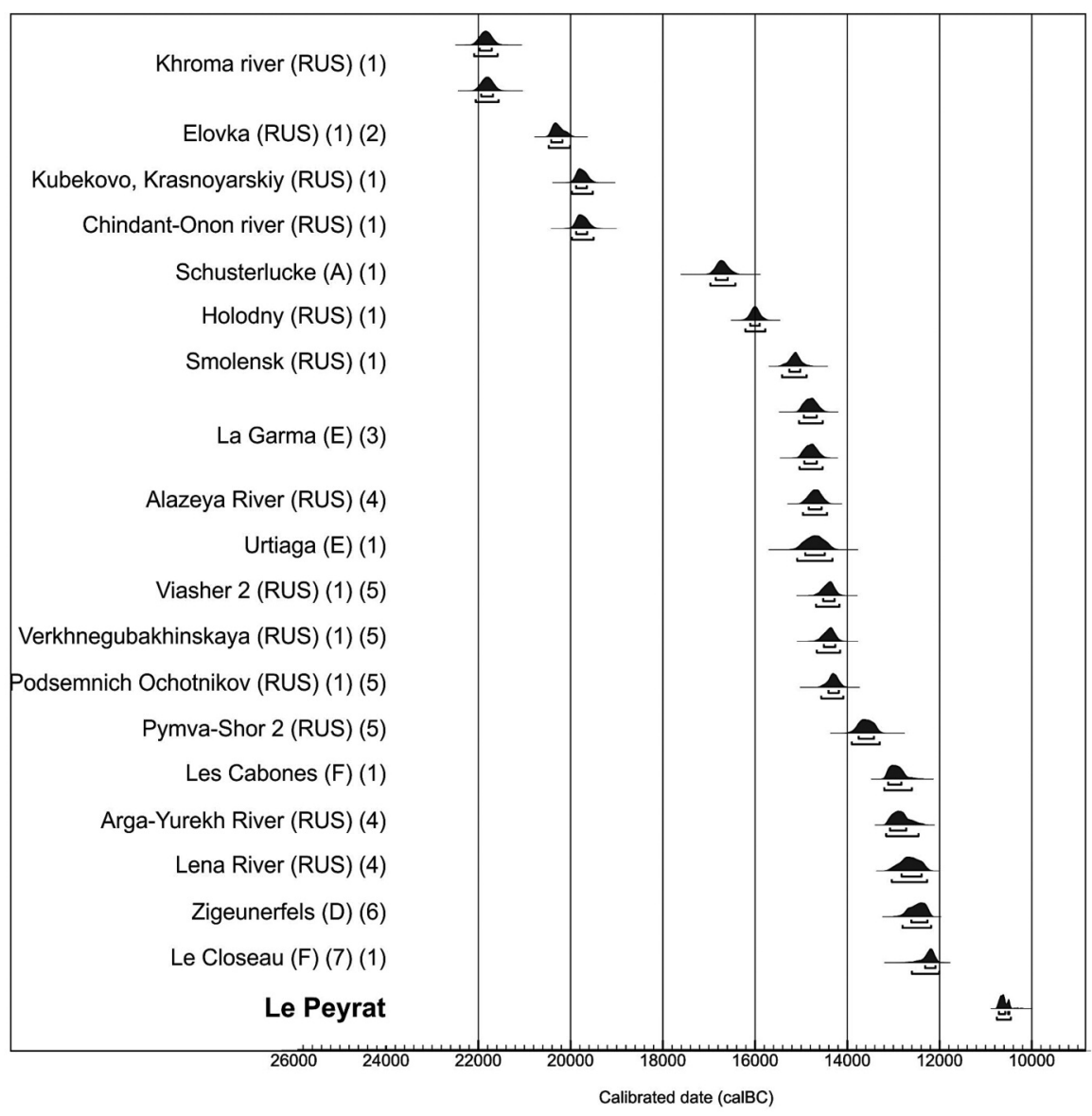

Figure 9 - Bilan des dates directes radiocarbone calibrées $(2 \sigma)$ sur os de lion des cavernes pour toute l'Eurasie. Source : (1) Stuart \& Lister, 2011 ; (2) Shchetnikov et al., 2015 ; (3) Cueto et al., 2016 ; (4) Barnett et al., 2009 ; (5) : Kosintsev et al., 2016 ; (6) Fahlke, 2009 ; (7) Bodu \& Mevel, 2008. $A=$ Autriche $; D=$ Allemagne ; $E=$ Espagne $; F=$ France ; RUS = Russie.

Figure 9 - A survey from all calibrates direct radiocarbon dates $(2 \sigma)$ for the cave lion from Eurasia. Source: (1) Stuart \& Lister, 2011; (2) Shchetnikov et al., 2015; (3) Cueto et al., 2016; (4) Barnett et al., 2009; (5) : Kosintsev et al., 2016; (6) Fahlke, 2009; (7) Bodu \& Mevel, 2008 $A=$ Austria ; $D=$ Germany $; E=$ Spain ; $F=$ France $;$ RUS $=$ Russia.
13000 ans cal BC (berges des rivières Arga-Yurekh : 12525 \pm 50 et de la Léna : $12450 \pm 60$; Barnett et al. 2009, soit respectivement $-13157 /-12456$ et $-13036 /-12270$ cal BC $(2 \sigma)$.

Dans le Nord-Ouest de l'Espagne, les lions les plus récents à ce jour sont uniformément datés, de $13770 \pm 120$ uncal BP (couche E de Urtiaga : Stuart \& Lister 2011) et de $13830 \pm 55$ uncal BP (paléosol de La Garma : Cueto et al. 2016), soit -15000 cal BC et sont attribués au Magdalénien moyen. Pour l'Allemagne, la date directe la plus récente (12375 \pm 50 uncal BP, soit $-12803 /-12184$ cal BC) provient de la couche $F$ (Fahlke 2009 : 27) ou « sans indication » (Fahlke 2009 : 269, repris par Stuart \& Lister 2011) de Zigeunerfels et est rattachée à un Magdalénien I.s.. Dans cette grotte, aucune attribution chrono-culturelle antérieure au Magdalénien n'a été relevée et la date obtenue sur une canine de lion des cavernes est la plus ancienne des 13 autres dates radiocarbone directes, couvrant l'ensemble de la stratigraphie (Fahlke, ibid). En France, deux dates radiocarbone, sub-synchrones, couvrent la période chronologique retenue. L'abri des Cabônes (ou abri Ranchot), fouillé au moins dans les années 1950 a livré une industrie magdalénienne (Magdalénien IV, VI) et azilienne, surmontée de couches mésolithiques. Un reste de lion est rapporté au Magdalénien I.s. (David 1996 : 181, tableau IX) et une date de $12565 \pm 50$ uncal BP a été obtenue (Stuart \& Lister 2011), soit -13199/-12600 cal BC. Aucune précision n'est portée sur la canine de lion alors même que le grand félidé est souvent mentionné « panthère ». Enfin, dans le locus 46 du Closeau, un métacarpe $V$ de lion trouvé avec 11 autres restes du même individu dans un Azilien ancien, est daté de $12248 \pm 70$ uncal BP (Bodu \& Mevel 2008), soit -12300 cal BC. Stuart \& Lister (2011) reprennent ce résultat, en mentionnant néanmoins qu'une nouvelle tentative de datation a échoué. Cet os du Closeau avait permis d'obtenir, avant l'analyse effectuée sur la vertèbre du Peyrat, la date la plus récente pour toute l'Eurasie puisque le spécimen de Lathum (Pays Bas), originellement daté de $10670 \pm 160$ uncal BP par AMS (Stuart \& Lister 2011 citant une référence relativement ancienne [Housley 1991]) a livré une nouvelle date de $44850 \pm 650$ uncal BP (Stuart \& List, op. cit.). Bien qu'échantillonné à une seule reprise, l'élément du Peyrat permet d'envisager la persistance du lion des cavernes presque 1500 ans de plus dans le Sud de l'Europe qu'auparavant, au moins jusqu'au début du Dryas récent car ce résultat est en accord avec le contexte chrono-culturel révisé, évoquant une industrie postérieure au Magdalénien et à l'Azilien décrit par A. Cheynier (Cheynier 1946b), c'est-à-dire l'actuel Azilien ancien (cf. supra). Cette date est même sensiblement plus jeune que toutes les datations effectuées sur des échantillons d'Amérique du Nord (Stuart \& Lister, ibid). Le schéma d'une disparition progressive en Eurasie, depuis le Sud vers le Nord et non antérieure à -12000 cal BC d'une part ainsi qu'une survivance plus tardive en Amérique du 
Nord que dans l'Ancien Monde d'autre part n'est peut-être plus évident.

\section{Le lion des cavernes entre 20000 et 10000 ans $\mathrm{BP}$ : les données chrono-culturelles}

Le lion des cavernes est particulièrement abondant au Paléolithique supérieur ancien (Aurignacien, en connexion avec son importance dans l'expression symbolique ?), puis se raréfie au cours du Gravettien et du Solutréen avant d'être à nouveau fréquent dans les différentes phases du Magdalénien (fig. 10). L'espèce est encore identifiée à l'Azilien et même dans des cultures du Postglaciaire (Asturien). Si l'identification du lion des cavernes au Paléolithique supérieur ne pose guère de difficultés pour de nombreux sites en France (Delpech 1975, 1983 ; Dufour 1989), il en va autrement pour attribuer et/ou valider précisément la chrono-culture dans laquelle l'espèce est identifiée, en raison de multiples écueils (échantillons paléontologiques non illustrés, non revus, ancienneté des fouilles, complexité de l'histoire taphonomique des remplissages, calibration rétrospective de dates radiocarbone conventionnelles, utilisation d'une date obtenue sur d'autres taxons, charbons ... pour caler un niveau ...). Un rapide aperçu des données et/ou contextes dans lesquels le lion des cavernes a été identifié dans le Sud de l'Europe fait ressortir toute l'importance du gisement du Peyrat.

Dans la grotte du Gros Roc (Charente-Maritime), quelques restes de lion des cavernes de petite taille (sans précisions ostéométriques) ont été identifiés dans un niveau magdalénien surmontant directement un ensemble moustérien (Clouet 1891). Ce lion a été nommé Panthera spelaea var. cloueti Boule 1906 pour signifier une différence de taille très nette par rapport aux grands lions des cavernes, courants au Pléistocène supérieur. Une étude sur la collection lithique (Daniel 1973) signale que la couche supérieure n'est pas magdalénienne mais aurignacienne ; ces observations sont beaucoup plus en accord avec le cortège faunique identifié dès la fin du $\mathrm{XIX}^{\ominus}$ siècle (présence concomitante de l'hyène des cavernes et de l'ours des cavernes dans ce même niveau). Cette reconsidération chrono-culturelle est particulièrement intéressante pour confirmer, une fois de plus, que les différences de taille relevées chez les lions des cavernes reflètent avant tout un important dimorphisme sexuel et non une attribution chronologique précise.

Dans la grotte des Scilles (Haute-Garonne), une M/1droite et une P/4 gauche présentent à la base de leurs racines plusieurs rainures profondes, interprétées comme le résultat de sciages. Ces dents, proviennent du niveau $B$, attribué à un Magdalénien « inférieur » (Saint-Périer 1926 : $21,27)$. Un bilan pluridisciplinaire récent de ce niveau archéologique confirme un âge ancien (16180 \pm 140 uncal $\mathrm{BP}$ ) et une faune composée de renne, cheval, chamois, saïga (Langlais et al. 2010).

Dans l'important gisement del Juyo, dont l'ensemble du remplissage est attribué au Magdalénien inférieur cantabrique, un seul reste de lion des cavernes a été identifié et provient du niveau 9. Les occupations humaines s'échelonnent entre 15000 et 13000 uncal BP (Klein \& Cruz-Uribe 1987).

À la grotte de La Vache (Ariège), une phalange II est mentionnée (Pailhaugue, 1995, 2003 : 78-79 ; même pièce déjà signalée par Garrigou 1867 : 60 ?) mais non étudiée précisément. Cette pièce paléontologique figure parmi un cortège faunique largement dominé par le bouquetin, pour lequel quelques datations $\mathrm{C} 14$, effectuées sur esquilles osseuses, sont comprises entre 14000 et 13000 ans uncal BP.

Dans le gisement du Morin (Gironde), plusieurs restes de lion des cavernes ont été identifiés, dans le niveau $A$, attribué au Magdalénien supérieur ( $\mathrm{VI})$ : une phalange I distale en AIII et des métacarpes II, III, IV gauches, un métacarpe $\mathrm{V}$ droit et un scapholunaire en AIV (Delpech, 1967 : 127) ainsi qu'une I3/ toujours en AIV et 1 i/3 (Delpech $1975:$ 257) et une phalange II (Delpech, 1983 : 231) en All. Ces vestiges doivent être synchrones des restes de chien identifié en Alll et directement datés de 12500 uncal BP (Boudadi-Maligne et al., 2012 : 48). Les mesures relevées sur les métacarpes témoignent, sans exception, d'individus plus petits que les lionnes de Jaurens ou encore d'Azoleta.

Dans la grotte du Bois du Cantet, une faune froide, dominée par le renne a été identifiée et renfermait dans le locus « boyau 7 » une carnassière inférieure de lion des cavernes (Clot et al. 1984). Une date radiocarbone (conventionnelle, sur esquilles) a donné 10700 uncal BP. La carnassière de lion des cavernes est de petite taille. Non revu, ce gisement pourrait être relativement contemporain de la grotte du Peyrat.

Dans la grotte de La Tourasse (Haute-Garonne), Harlé (1894 : 403) a identifié cinq restes de lion des cavernes (une canine supérieure déciduale, un fragment distal de métapode, un phalange I et deux canines définitives) dans un niveau azilien, en association avec le cerf, un grand bovidé, le cheval et le sanglier. Aucune illustration ni donnée ostéométrique ne figure dans la présentation de Harlé. Dans les collections du Muséum d'histoire naturelle de Toulouse, la faune de La Tourasse comporte principalement du renne et de l'antilope saïga. Aucun vestige de lion des cavernes n'a été vu. De plus, Dufour (1989 : 135) ne signale pas la présence du félin dans cette station parmi les collections régionales « Harlé » du Muséum d'histoire naturelle de Bordeaux. La composition faunique (abondance de l'antilope saiga) s'il n'y a pas eu d'erreur d'étiquetage des collections d'une part, et la " disparition » de ces restes paléontologiques d'autre part ne permettent pas objectivement d'assurer la présence du lion dans ce gisement ou, localement, à cette chronoculture (Azilien).

Dans la grotte multi-stratifiée de La Riera, des restes de lion des cavernes ont été signalés dans les niveaux supérieurs du remplissage, à savoir les couches 27 (Magdalénien 

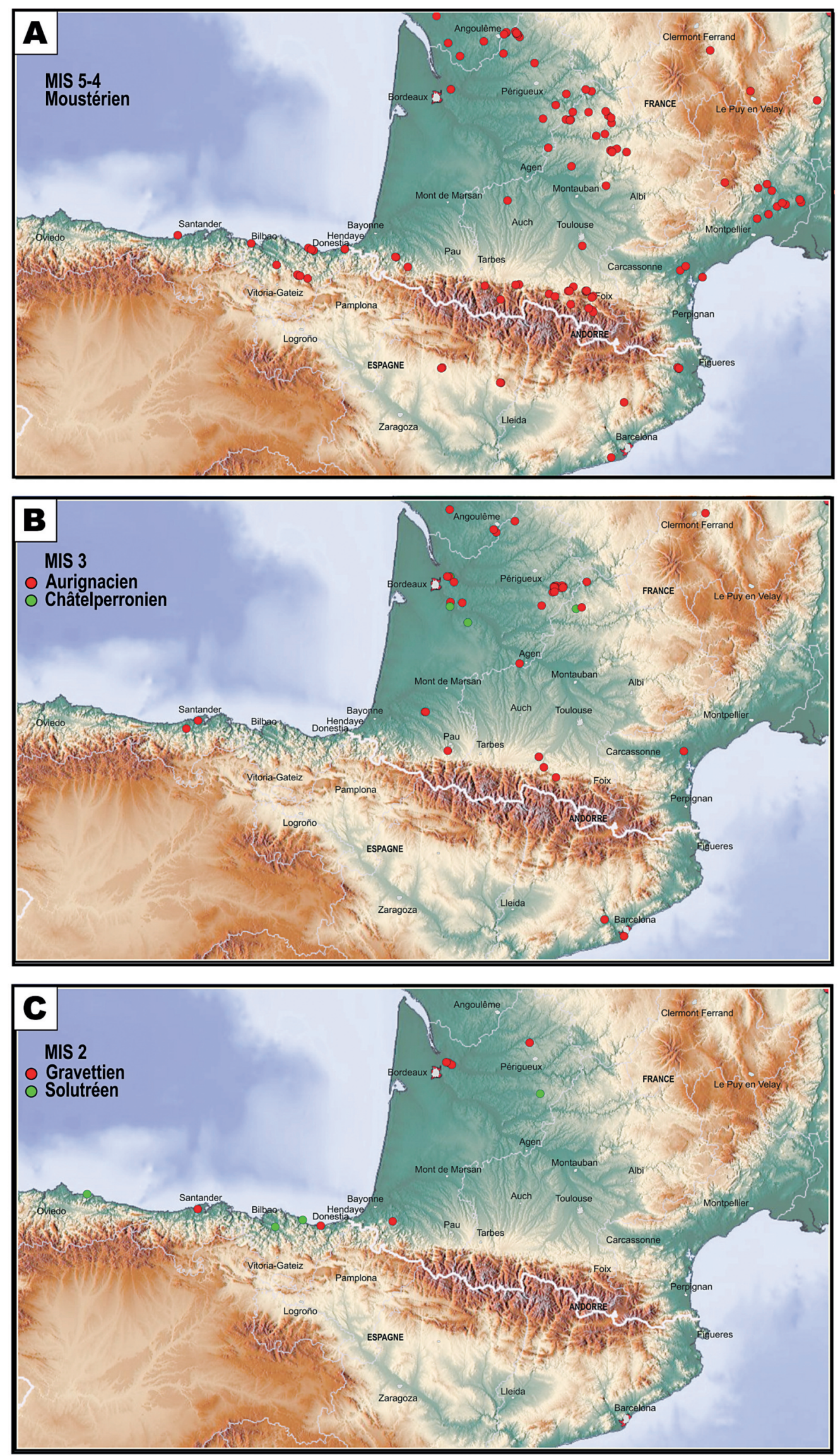

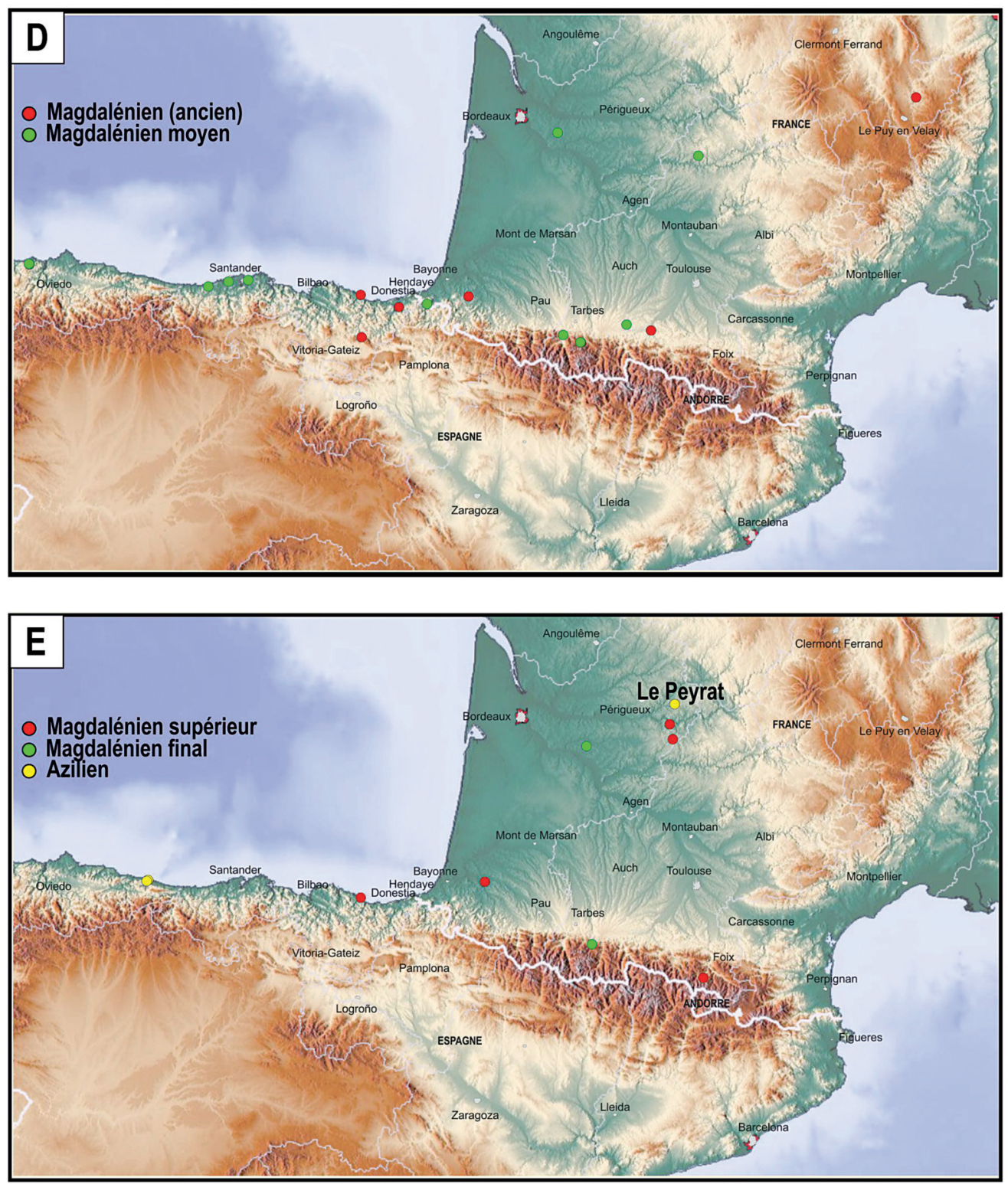

Figure 10 - Répartition géo-chronologique du lion des cavernes dans le Sud-Ouest de la France, les Pyrénées et le Nord-Ouest de l'Espagne au Pléistocène supérieur. A = MIS 5-4 et Moustérien ; $B=$ MIS 3, Châtelperronien et Aurignacien/Chatelperronien ; $C=M I S$ 2, Gravettien et Solutréen ; $D=$ Magdalénien, Magdalénien ancien et Magdalénien moyen ; $E$ = Magdalénien supérieur, Magdalénien final et Azilien. voir Annexe pour liste des gisements.

Figure 10 - Geo-chronological distribution of cave lion in Southwestern France, Pyrenees and Northwestern Spain during Late Pleistocene. $A=$ MIS 5-4 and Mousterian ; $B=$ MIS 3, Chatelporronian and Aurignacian; $C=$ MIS 2, Gravettian and Solutrean; $D=$ Magdalenian, Lower Magdalenian and Middle Magdalenian ; $E=$ Late Magdalenian, Latest Magdalenian and Azilian. See Appendice for sites drawn on maps. 
final/Azilien) et 29 (Mésolithique ancien ?/Asturien). La faune est, dans toutes les séquences, dominée par le cerf et le bouquetin ibérique (Altuna 1986). Des dates radiocarbone conventionnelles rattachent le niveau 27 à l'Allerod et le niveau 29 à l'Holocène, au Préboréal/Boréal. Les dimensions modestes des restes ont potentiellement évoqué le lion d'Afrique (Altuna, ibid). Aucune révision stratigraphique, aucune date AMS, aucune étude paléogénétique n'est disponible pour ces restes paléontologiques. Sommer \& Benecke (2006: 14) soulignent qu'en raison de la variabilité intraspécifique chez les Félidés, il est difficile de rapporter ces quelques restes isolés à l'une ou l'autre des deux espèces d'une part et que le lion africain n'est identifié en Europe qu'à partir de la chronozone Atlantique d'autre part.

Récemment, Masseti \& Mazza (2013) présentent quatre gisements/niveaux en Italie renfermant des restes de lion des cavernes, avec des dates comprises entre 12000 et 9000 BP. Dans le niveau $L$ de la grotta della Madonna, des charbons de bois et des esquilles osseuses brûlées ont donné $12100 \pm 150$ et $9020 \pm 125$ (dates non calibrées ?). À Riparo Fredian, le niveau épigravettien est daté de $10870 \pm 119$ et le niveau 4 de $9458 \pm 91$ (charbons de bois). Enfin, à la grotta della Onda, une date U/Th obtenue sur calcite donne $10710 \pm 200$ (Masseti \& Mazza, op. cit.). Bien que toutes ces dates soient livrées sans numéro de laboratoire et avec des références bibliographiques anciennes (années 1960-1990) et bien que Stuart \& Lister (2011) ne retiennent aucune date radiométrique pour les sites italiens, il ressort que le lion des cavernes pourrait avoir perduré en Italie au moins aussi longtemps que dans le Sud-Ouest de la France. À ce jour, le gisement du Peyrat constitue la seule évidence vérifiée de la survivance du lion des cavernes jusqu'à l'extrême fin du Paléolithique et confirme la deuxième hypothèse de recherche de Masseti \& Mazza (op. cit.) évoquant la présence possible de l'espèce jusqu'au début du Postglaciaire dans le Sud de l'Europe. Les lions modernes (Panthera (Leo) persica) ne sont identifiés dans le Sud-Est de l'Europe (Bulgarie, Hongrie ...) qu'à partir de l'Age du Cuivre et à l'Age du Bronze (Vörös 1983 ; Nobis \& Ninov 2002 ; Benecke \& Sommer, op. cit. ; Németh et al. 2017). Un hiatus d'environ 5000 ans sépare la disparition du lion des cavernes et l'apparition du lion africain.

\section{L'exploitation du lion des cavernes au Paléolithique : les données archéozoologiques}

Bien qu'identifié dans près d'un millier de gisements/niveaux tout au long du Pléistocène moyen et supérieur, le lion des cavernes I.s. a été, à l'instar des autres grands Carnivores, rarement chassé et/ou exploité au cours des temps paléolithiques. Une dizaine de mentions environ rapporte des traces d'origine anthropique sur des restes de lion des cavernes, sous la forme de stries de découpe.

La plus ancienne preuve d'un traitement anthropique de carcasse de lion des cavernes provient d'Atapuerca (Gran Dolina) et est attribuée au Pléistocène moyen récent (MIS 9) au travers d'une extrémité proximale de radius
(Blasco et al. 2010). Toutes les autres observations peuvent être rapportées au Pléistocène supérieur. Dans la grotte à ours des cavernes de Fate (Italie), une phalange II portant des stries a été recueillie dans un niveau moustérien (Valensi \& Psathi 2004). Dans le site fluviatile de Siegsdorf (Allemagne), un squelette complet de lion, daté de plus de 45000 ans (Stuart \& Lister 2011), a été retrouvé. Si les éléments squelettiques sont presque tous complets (Gross 1992), des traces de décarnisation ont été parfaitement identifiées sur un fémur et un tibia droits, dans un contexte également Moustérien (Gross, ibid). Les évidences d'exploitation du lion des cavernes sont moins rares pour le Paléolithique supérieur que pour les périodes précédentes ; à Arcy-sur-Cure (grotte du Renne), une phalange II, provenant d'un niveau châtelperronien (Madelaine \& Fosse, observations personnelles) présente de nombreuses stries jalonnant toute la longueur diaphysaire des faces médiale et latérale. Dans des horizons culturels sensiblement contemporains (Aurignacien/Gravettien), dans la grotte de Hohle Fels (Allemagne), un métacarpe et un tibia complets présentent des traces de découpe ; dans un niveau gravettien, une diaphyse d'ulna a également été retrouvée (Conard \& Münzel, com. pers.). Au Magdalénien (moyen), sur un paléosol dans la grotte de La Garma (Espagne), 9 phalanges III présentent de nombreuses traces de découpe (Cueto et al. 2016). Dans la « grotte des Eyzies » [= grotte Richard, Magdalénien récent], Lartet \& Christy (1864 : 21) signalent une pièce exceptionnelle qui est « un métacarpien de petit doigt d'un jeune felis de très grande taille ( $F$. spelaea ?), qui présente des traces nombreuses d'entailles et de rayures, absolument de la même façon que les os des autres animaux mangés par les aborigènes ". Enfin, dans le site de plein air du Closeau, 12 restes de lion (humérus distal, métacarpes, métatarses), fracturés, brûlés et sciés proviennent du locus 46 parmi une faune dominée par le cheval et le cerf (Bémilli 2000). Les vertèbres découpées du Peyrat complètent cette courte liste des gisements renfermant des indices d'exploitation du lion des cavernes. À l'exception du Closeau qui traduit une consommation effective du lion, les sites reflètent principalement des activités de dépouillement, afin de récupérer la fourrure ; os dépourvus d'intérêt alimentaire (vertèbres, métapodes, phalanges) et os longs complets (fémurs, tibias) constituent les observations les plus fréquentes d'exploitation du lion des cavernes au Paléolithique.

\section{Conclusions}

La révision sommaire de la grande faune ainsi que des séries lithiques et osseuses du gisement du Peyrat a permis d'identifier le lion des cavernes dans des niveaux plus récents que le Magdalénien supérieur auquel ces restes osseux avaient été initialement rattachés. Cette reconsidération culturelle est confirmée par une date radiocarbone directe. II apparaît que le lion des cavernes a perduré, dans le Sud de l'Europe, au moins jusqu'au Dryas récent, contemporain de l'Azilien récent. La grotte du Peyrat constitue le seul gisement d'Aquitaine renfermant du lion des cavernes pour cette période, le grand Carnivore identifié dans les sites archéologiques contemporains étant 
le loup. L'exploitation d'un grand prédateur est, dans ce gisement du Tardiglaciaire, effective sans que le rôle des groupes humains dans l'extinction d'une espèce animale puisse être abordée. Enfin, cette révision ostéologique et chrono-culturelle démontre tout l'intérêt d'une approche pluridisciplinaire de collections muséographiques et participe activement aux débats sur l'extinction de la grande faune glaciaire dans le Sud de l'Europe.

\section{Remerciements}

Les auteurs adressent leurs remerciements les plus sincères auprès des Institutions et personnes ayant facilité l'accès aux collections ou contribué à ce travail par l'apport de données, à savoir : Jean-Jacques Cleyet-Merle, MarieDominique Dehe, Philipe Jugie du Musée National de Préhistoire (Les Eyzies-de-Tayac), Catherine Schwab du Musée d'Archéologie nationale (Saint-Germain-en-Laye), Henri Cap, Pierre Dalous, Francis Duranthon, Guillaume Fleury du Muséum d'Histoire naturelle de Toulouse, Evelyne Crégut (Avignon), Jean-Baptiste Fourvel (Toulouse), Nicolas Frèrebeau (Bordeaux \& Saclay), Alain du Fayet de La Tour (Foissac), Pedro Castaños (Bilbao), Nicholas Conard \& Suzanne Münzel (Tübingen). Les auteurs remercient également les relecteurs qui ont permis d'améliorer ce travail, ainsi que Brad Gravina pour la traduction de l'abstract. La date radiocarbone a été effectuée dans le cadre d'un projet Artemis.

\section{Références bibliographiques}

ALTUNA J. 1972 - Fauna de mamiferos de los yacimientos prehistoricos de Guipuzcoa. Munibe, 24(1-4) : 1-464.

ALTUNA J. 1981 - Fund eines skeletts des Höhlenlöwen (Panthera spelaea Goldfuss) in Arrikrutz, Baskenland Bonner zoologische Beiträge, 32(1-2) : 31-45.

ALTUNA J. 1986 - The mammalian faunas from the prehistoric site of la Riera. In : L.G. Straus \& G. Clark (eds), La Riera Cave : 237-274, 421-480.

ARGANT A. 1991 - Carnivores quaternaires de Bourgogne. Doc. Lab. Géol. Lyon,115 : 1-301.

ARGANT A. 2010 - Carnivores (Canidae, Felidae et Ursidae) de Romain-la-Roche (Doubs, France). Revue de Paléobiologie, 29(2) : 495-601.

ARRIBAS O. 2004 - Fauna y paisaje de los Pirineos en la era glaciar. Lynx edicions : 1-540.

BALLÉSIO R. 1980 - Le gisement pléistocène supérieur de la grotte de Jaurens à Nespouls, Corrèze, France : les Carnivores : II Felidae. Nouvelles Archives du Museum d'Histoire naturelle de Lyon, 18 : 61-102.

BARNETT R., SHAPIRO B., BARNES I., HO S.Y.W., BURGER J., YAMAGUCHI N., HIGHAM T.F.G., WHEELER H.T., ROSENDAHL W., SHER A.V., SOTNIKOVA M., KUZNETSOVA T., BARYSHNIKOV G.F., MARTIN L.D., HARINGTON C.R., BURNS J.A., COOPER A. 2009 -
Phylogeography of lions (Panthera leo ssp.) reveals three distinct taxa and a late Pleistocene reduction in genetic diversity. Molecular Ecology, 18 : 1668-1677.

BARNETT R., LISANDRA M., ZEPEDA MENDOZA M.L., ELIAS A., SOARES R., HO S.Y.W., ZAZULA G., YAMAGUCHI N., SHAPIRO B., KIRILLOVA I., LARSON G., THOMAS M., GILBERT P. 2016 - Mitogenomics of the extinct cave lion, Panthera spelaea (Goldfuss, 1810) resolve its position within the Panthera cats. Open Quaternary, 2(4): 1-11.

BARONE R. 1976 - Anatomie comparée des mammifères domestiques. Tome 1 : ostéologie (fasc. 1, texte; fasc. 2, atlas). Paris, Vigot Frères.

BARYCKA E. 2008 - Middle and Late Pleistocene Felidae and Hyaenidae of Poland. Fauna Poloniae, New Series, 2 : $1-228$.

BÉMILLI C. 2000 - Nouvelles données sur les faunes aziliennes du Closeau, Rueil-Malmaison (Hauts-de-Seine). Bulletin de la Société Préhistorique Française, XXVIII : 29-38.

BLASCO R., ROSELL ARDÉVOL J., ARSUAGA FERRERAS J.L., BERMUDEZ DE CASTRO J.M., CARBONELL ROURA E. 2010 - The hunted hunter: the capture of a lion (Panthera leo fossilis) at the Gran Dolina site, Sierra de Atapuerca, Spain. Journal of archaeological Science, 37(8) : 2051-2060.

BODU P., MEVEL L. 2008 - Enquête autour des lames tranchantes de l'Azilien ancien. Le cas du niveau inférieur du Closeau (Rueil-Malmaison, Hauts-de-Seine, France). L'Anthropologie, 112 : 509-543.

BONIFAY M.F. 1971 - Carnivores quaternaires du Sud-Est de la France. Museum national d'Histoire naturelle (Paris), série C, Mémoires, 21(2) : 43-377.

BOUDADI-MALIGNE M., MALLYE J.B., LANGLAIS M., BARSHAY-SZMIDT C. (2012) - Des restes de chiens magdaléniens à l'abri du Morin (Gironde, France). Implications socio-économiques d'une innovation zootechnique. Paleo, 23 : 39-54.

BOULE M. 1906 - Les grands chats des cavernes. Annales de Paléontologie, 1 : 1-27.

BRONK RAMSEY C. 2009 - Bayesian analysis of radiocarbon dates. Radiocarbon, 51(1) : 337-360.

CASTAÑOS P. 2005 - Estudio paleontológico de un esqueleto de león (Panthera leo) de la sima de Azoleta (Gorbeia, Alava). Munibe, 57 : 123-129.

CHEYNIER A. 1964a - La baguette gravée magdalénienne du Peyrat, Bulletin de la Société Historique et Archéologique du Périgord, 91 : 25-27.

CHEYNIER A. 1964b - Les fouilles du Peyrat, campagne 1964, Bulletin de la Société Préhistorique Française, 61 : 169-170 (communication). 
CLEYET-MERLE J.-J. 1988 - Le gisement magdalénien du Peyrat à Saint-Rabier (Dordogne) d'après les fouilles Cheynier, 1958-1967. Bulletin de la Société Préhistorique Française, 85(10-12) : 332-351.

CLOT A. 1980 - La grotte de la Carrière (Gerde, HautesPyrénées). Stratigraphie et Paléontologie des Carnivores. Thèse Université Paul Sabatier Toulouse : 237 p.

CLOT A., BROCHET G., CHALINE J., DESSE J., EVIN J., GRANIER J., MEIN P., MOURER-CHAUVIRÉ C., OMNĖS J., RAGE J.C. 1984 - Faune de la grotte préhistorique du bois du Cantet (Espèche, Hautes Pyrénées, France). Munibe, 36 : 33-50.

CLOT A., DURANTHON F. (1990) - Les Mammifères fossiles du Quaternaire dans les Pyrénées. Toulouse, $150 \mathrm{p}$.

CLOUET 1891 - Fouille de la grotte du Gros-Roc, commune du Douhet (Charente inférieure). AFAS.

COSTAMAGNO S., DURANTHON F., ROUZAUD F., DETRAIN L., DUFOUR P., ANTOINE P.O. 1997 - L'Aven du Lion (lieu-dit Bramefond commune de Souillac, Lot). D.F.S. de sauvetage urgent : 1-36

CUETO M., CAMARÓS E., CASTAÑOS P., ONTAÑÓN R., ARIAS P. 2016 - Under the skin of a lion: unique evidence of Upper Paleolithic exploitation and use of cave lion (Panthera spelaea) from the Lower Gallery of La Garma (Spain). PLoS ONE, 11(10): e0163591.

DANIEL R. 1973 - Présentation d'une série lithique de la grotte du «Gros-Roc », commune du Douhet (CharenteMaritime) provenant des fouilles Clouet (1889). Bulletin de la Société préhistorique française, 70(3) : 80-84.

DAVID S. 1996 - La fin du Paléolithique supérieur en Franche-Comté : environnement, cultures, chronologie. Gallia préhistoire, 38 : 111-248.

DELPECH F. 1967 - Recherches paléontologiques concernant quelques gisements du Magdalénien VI : stations de la Gare de Couze (Dordogne), du Morin (Gironde) et de Duruthy (Landes). Thèse $3^{\mathrm{e}}$ cycle Université de Bordeaux I.

DELPECH F. 1975 - Les faunes du Paléolithique supérieur dans le Sud-Ouest de la France. Thèse Université Bordeaux I.

DELPECH F. 1983 - Les faunes du Paléolithique supérieur dans le Sud-Ouest de la France. Cahiers du Quaternaire, $6: 453 \mathrm{p}$.

DESSE J., CHAIX L., DESSE-BERSET N. 1986 “ Ostéo »; base-réseau de données ostéométriques pour l'archéozoologie. Procédures, codages, exploitation des données, organisation du réseau. CRA, Notes et Monographies techniques, $20: 161$.
DIEDRICH C.G., RATHGEBER T. 2012 - Late Pleistocene steppe lion Panthera leo spelaea (Goldfuss 1810) skeleton remains of the Upper Rhine Valley (SW Germany) and contributions to their sexual dimorphism, taphonomy and habitus. Historical Biology: An International Journal of Paleobiology, 24 (1) : 1-28.

DIETRICH W.O. 1968 - Fossile Löwen im Europäischen und Afrikanischen Pleistozän. Paläont. Abh., A III(2) : 323-366.

DRIESCH VON DEN A. 1976 - A guide to the measurement of animal bones from archaeological sites. Peabody Museum Bulletins, 1 : 1-137.

DUFOUR R. 1989 - Les Carnivores pléistocènes de la caverne de Malarnaud (Ariège). D.E.S. Université de Bordeaux I : $456 \mathrm{p}$.

FAHLKE M. 2009 - Der Austausch der terrestrischen Säugetierfauna an der Pleistozän/Holozän-Grenze in Mitteleuropa. phD Fakultät der Rheinischen FriedrichWilhelms Universität Bonn : 279 p.

FILHOL, H. FILHOL M. 1871 - Description des éléments de Felis spelaea découverts dans la caverne de l'Herm, Ariège. Ann. Sciences Nat., 5ème série, XIV: 1-120.

GARRIGOU F. 1867 - Age du Renne dans la grotte de la Vache (Vallée de Niaux), près de Tarascon (Ariège). Bulletin de la Société d'Histoire naturelle de Toulouse, 1 : 58-67.

GROSS C. 1992 - Das skelett des Höhlenlöwen (Panthera leo spelaea Goldfuss, 1810) aus Siegsdorf/Ldkr. Traunstien im Vergleich mit anderen Funden aus Deutschland und den Niederlanden. phD Institut für Palaeoanatomie, Domestikationforschung und Geschichte der Tiermedizin, Universität München : $128 \mathrm{p}$.

HARLÉ E. 1894 - Restes d'Elan et de Lion dans une station préhistorique de transition entre le Quaternaire et les temps actuels à Saint-Martory (Haute Garonne). L'Anthropologie, IV : 402-406.

HEMMER H. 2011 - The story of the cave lion Panthera leo spelaea (Goldfuss, 1810) : a review. Quaternaire, Horssérie(4) : 201-208.

KLEIN R.G., CRUZ-URIBE K. 1987 - La fauna mamifera del yacimiento de la cueva de «El Juyo » campanas de 1978 y 1979 . Centro de Investigacion y Museo de Altamira, Monografias, 14 : 99-120.

KOSINTSEV P.A., GASILIN V.V., GIMRANOV D.O., BACHURA O.P. 2016 - Carnivores (Mammalia, Carnivora) of the Urals in the Late Pleistocene and Holocene. Quaternary International, 420 : 145-155.

KROFEL M. 2004 - A find of Panthera leo spelaea GOLDFUSS (Felidae, Mammalia) from unknown stratigraphic position of Poto ka zijalka (Slovenia). Mitt. Komm. Quartärforsch. Österr. Akad. Wiss., 13 : 81-88. 
KURTEN B. 1985 - The Pleistocene lion of Beringia. Ann. Zool. Fennici, 22 : 117-121.

LANGLAIS M., PÉTILLON J.M., BEAUNE (DE) S.A., CATTELAIN P., CHAUVIĖRE F.X., LETOURNEUX C., SZMIDT C.C., BELLIER C., BEUKENS R., DAVID F. 2010 - Une occupation de la fin du Dernier Maximum glaciaire dans les Pyrénées : le Magdalénien inférieur de la grotte des Scilles (Lespugue, Hautes-Pyrénées). Bulletin de la Société préhistorique française, 107(1) : 5-51.

LARTET L., CHRISTY H. 1864 - Cavernes du Périgord. Objets gravés et sculptés des temps pré-historiques dans l'Europe occidentale. Paris, $37 \mathrm{p}$

LARTET L., CHAPLAIN DUPARC C. 1874 - Une sépulture des anciens troglodytes des Pyrénées superposée à un foyer contenant des débris humains associés à des dents sculptées de lion et d'ours. Paris, Masson : $67 \mathrm{p}$.

MARCISZAK A., STEFANIAK K. 2010 - Two forms of cave lion: Middle Pleistocene Panthera spelaea fossilis REICHENAU, 1906 and Upper Pleistocene Panthera spelaea spelaea GOLDFUSS, 1810 from the Bisnik Cave, Poland. N. Jb. Geol. Paläont. Abh., 258(3) : 339-351.

MASSETI M., MAZZA P.A. 2013 - Western European Quaternary lions: new working hypotheses. Biological Journal of the Linnean Society, 109 : 66-77.

MEACHEN-SAMUELS J.A., BINDER W.J. 2009 - Sexual dimorphism and ontogenetic growth in the American lion and sabertoothed cat from Rancho La Brea. Journal of Zoology, 280 : 271-279.

MERRIAM J., STOCK C. (1932) - The Felidae of Rancho La Brea. Carnegie Institution of Washington. Publication Number 422, 231p.

NÉMETH A., BÁRÁNY A., CSORBA G., MAGYARI E., PAZONYI P. PÁLFY J. 2017 - Holocene mammal extinctions in the Carpathian Basin : a review. Mammal Review, 47(1): 38-52.

NOBIS G., NINOV L. (2002) - Zur Fauna der prähistorischen Siedlung Durankulak, Bez. Tolbuchin (NOBulgarien). II. Die Kupferzeit. Bonner zoologische Monographien, 51 : 29-59.

PACHER M. 2009 - Funde des Höhlenlöwen (Panthera leo spelaea) aus der Herdengelhöhle (1823/4) bei Lunz am See, Niederösterreich. Die Höhle, 60 (1-4) : 21-27.

PAILHAUGUE N. 1995 - La faune de la salle Monique, Grotte de la vache (Alliat, Ariège). Bulletin de la Société Préhistorique de l'Ariège, L : 225-289.

PAILHAUGUE N. 2003 - La faune de la Salle Monique. In Clottes J., \& Delporte H., (dir.), La grotte de La Vache (Ariège). Tome l- Les occupations du Magdalénien. Musée des Antiquités Nationales, CTHS Editions (Paris) : 73-139.
PHILIPPE M., ARGANT A., ARGANT J. 2010 - Un programme d'inventaire, de datations et de données paléoenvironnementales concernant les grottes à ossements fossiles du massif de Chartreuse. 7èmes Rencontres du Patrimoines scientifique en Rhône-Alpes ; patrimoine naturel : le patrimoine géologique. Muséum d'histoire naturelle de Grenoble : 37-44.

REIMER P.J., BARD E., BAYLISS A., BECK J.W., BLACKWELL P.G., BRONK RAMSEY C., BUCK C.E., CHENG H., EDWARDS R.L., FRIEDRICH M., GROOTES P.M., GUILDERSON T.P., HAFLIDASON H., HAJDAS I., HATTÉ C., HEATON T.J., HOFFMANN D.L., HOGG A.G., HUGHEN K.A., KAISER K.F., KROMER B., MANNING S.W., NIU M., REIMER R.W., RICHARDS D.A., SCOTT E.M., SOUTHON J.R., STAFF R.A., TURNEY C.S.M., VAN DER PLICHT J. 2013 - IntCal13 and Marine13 Radiocarbon Age Calibration Curves 0-50,000 Years cal BP. Radiocarbon, 55(4) : 1869-1887.

SAINT-PÉRIER R. 1926 - La grotte des Scilles à Lespugue (Haute-Garonne). L'Anthropologie, XXXVI : 15-40.

SALA B. 1990 - Panthera leo fossilis (V. Reich.1906) (Felidae) de Isernia La Pineta (Pléistocène moyen inférieur d'Italie). Geobios, 23(2) : 189-194.

SAMSEL M., KNÜSEL C.J., VILLOTTE S. 2016 Réévaluation du sexe et de l'âge au décès du sujet azilien Le Peyrat 5, Saint-Rabier (Dordogne, France). Bulletin de la Société d'Anthropologie de Paris, 28 : 213-220.

SANCHIS A., TORMO C., SAUQUÉ V., SANCHIS V., DÍAZ R., RIBERA A., VILLAVERDE V. 2015 - Pleistocene leopards in the Iberian Peninsula: New evidence from palaeontological and archaeological contexts in the Mediterranean region. Quaternary Science Reviews, 124 : 175-208.

SCHÜTT G. (1969) - Untersuchungen am Gebiss von Panthera leo fossilis (v. REICHENAU 1906) und Panthera leo spelaea (GOLDFUSS 1810). Ein beitrag zur Systematik der pleistozänen Grosskatzen Europas. N. Jb. Geol. Paläont. Abh., 134(2) : 192-220.

SHCHETNIKOV A.A., KLEMENTIEV A.M., FILINOV I.A., SEMENEY E.Y. 2015 - Large Mammals from the Upper Neopleistocene reference sections in the Tunka Rift Valley, Southwestern Baikal region. Stratigraphy and Geological Correlation, 23(2): 214-236.

SOMMER R.S., BENECKE N. (2006) - Late Pleistocene and Holocene development of the felid fauna (Felidae) of Europe: a review. Journal of Zoology, 269 : 7-19.

SOTNIKOVA M., NIKOLSKIY P. 2006 - Systematic position of the cave lion Panthera spelaea (Goldfuss) based on cranial and dental characters. Quarternary International, $142-143: 218-228$.

SOTNIKOVA M.V., FORONOVA I.V. 2014 - First Asian record of Panthera (Leo) fossilis (Mammalia, Carnivora, 
Felidae) in the Early Pleistocene of Western Siberia, Russia. Integrative Zoology, 9 (4) : 517-530.

STUART A.J., LISTER A.M. 2011 - Extinction chronology of the cave lion Panthera spelaea. Quaternary Science Reviews, 30 (176) : 2329-2340.

TSOUKALA E., GRANDAL D'ANGLADE A. 2002 - Système de mesures sur le squelette d'Ursidae. In : Tillet, T. \& Binford, L.R. (eds) L'Homme et l'Ours, ERAUL : 265-287.

TURNER A. 1984 - Dental sex dimorphism in european lions (Panthera Leo L.) of the Upper Pleistocene : palaeoecological and palaeoethological implications. Annales zoologica fennici, 21 : 1-8.
VALENSI P., PSATHI E. 2004 - Faunal exploitation during the Middle Palaeolithic in South-eastern France and Northwestern Italy. International Journal of Osteoarchaeology, $14: 256-272$.

VÖRÖS I. 1983 - Lion remains from the Late Neolithic and Copper age of the Carpathian basin. Folia Archaeologica, XXXIV : 33-50. 


\section{Annexe - liste des gisements reportés sur les cartes \\ Appendice - sites cited in maps}

MIS 5-4, Moustérien - Arbreda ; Artenac, ens. 3 c6, c8 ; Bourgeois-Delaunay, 9,10 ; Calmette, La ; chez Pinaud, 8-5 ; Clos du Prieur, Le ; Combe Capelle bas ; Combe Grenal, 3,35,51 ; Crouzade, La, 6-8 ; Gabasa 1, e, g ; Gatzarria, cj ; Hortus, L' ; Imanolen Arrobia ; Lezetxiki, VI, VII, VIII ; Noutaris I ; Pair non Pair, F-F' ; Portel, Le, B, C, F2, K ; Reclau Viver ; Regourdou, 2 ; Rochelot ; Sainte Anne I, J2c ; Suard, V-VI, VIII ; Toll, i, j ; Tournal, B/C, D1/D2 ; Unikoté I, 10-12 ; Unikoté II, niv Plan.

MIS 5-4 probablement (voire MIS 3 ?), mentions générales - Altamira ; Arrikrutz ; Aubert ; Azoleta ; Baio ; Balauzière, La ; Baume-Longue ; Baume Rousse ; Beyrède Jumet ; Bosc ; Bouichéta ; Bramefond ; Cajarc ; Cave aux Endives, La ; Champs Gaillards, Les ; Champs verts, Les ; Châteaubourg ; Condom ; Coustal, Le ; Ermitage, L' ; Fineau, La ; Foissac ; Font de Gaume ; Fontanet ; Frères Traversat ; Gargas, diverticule Régnault ; Goudou, Rave Gallery ; Gourdan, grotte murée ; grotte XVI ; Infernet ; Lestélas ; Lherm ; Lombrives ; Loubeau ; Malarnaud ; Mas d'Azil, Le, RD ; Massat sup. ; Mialet ; Montaigu le Blin ; Montgaudier ; Padirac ; Pech de l’Aze ; Pénélos, Las ; Pépi, La ; Pexoumbarayré ; Plumettes, Les ; Pondres ; Portel, Le, B,K ; Prajoux, Q ; Pras de Marrou ; Punta Lucero ; Roland ; Santimamiñe, IV ; Sartanette, La ; Seynes ; Soulabé ; Soute, La ; Torre ; Tour de Boulade ; Triangle ; Trou du Cluzeau ; Tuteil ; Verrerie, La ; Vilhonneur, grotte 1 (moulin).

MIS 3 - abric Romani, I ; Bourdette, gal I, 8 ; BourgeoisDelaunay, 4 ; Camiac ; Junqua ; Miguet ; Mothe, La.
Châtelperronien - Cottès, Les (Périgordien) ; Fées, Les (Châtelperron) ; Haurets ; Lavison ; Pair non Pair, E-D' inf ; Roc de Combe, 8.

Aurignacien - Aurignac ; Canyars ; Castanet, Aurig I ; Castillo, Le, 18 ; Cro-Magnon ; Ferrassie (ga), La, F (Aurig I), G/H, H' (Aurig III), I, J/K/L (Aurig ancien) ; Ferrassie (g), La ; gros Roc ; Hyènes, Les, ensemble 1, US $2 \mathrm{C}$; Jolias ; Lartet ; Pair non Pair, D', K ; Pataud, 11 ; Pendo, Le, VII ; Piage, Le, GI ; Pont Neuf ; Rochette, La, 5 ; Rois, Les, niveau B ; Ruth, Le (abri Pagès) ; Souquette, La ; Tarté.

Gravettien - Amalda, V ; Fourneau du Diable, terrasse inf, C. Aurig ; Isturitz ; Pair non Pair, 4, CB6-7, D ; Pendo, Le, V.

Solutréen - Arlanpe, II ; Balmori, 5 ; Roc de Combe Capelle ; Urtiaga, I.

Magdalénien - Aitzbitarte IV ; Aizkirri ; Cottier ; Isturitz.

Magdalénien ancien - Scilles, Les ; Urtiaga, F.

Magdalénien moyen - Abauntz, e ; Altamira, Magd. ; Aurensan inférieur ; Aurensan supérieur ; Bergerie des Quatre Chemins ; Espélugues, Les ; Garma, La ; Juyo, El, 8-9 (Magd III) ; Moulin Neuf, c2 ; Paloma, La, 4, 6, 8.

Magdalénien supérieur - Duruthy, 3 (Magd VI) ; Flageolet, Le, VII ; Madeleine, La ; Urtiaga, E (Magd V ?) ; Vache, La, salle Monique.

Magdalénien final - Bois du Cantet, boyau 7 ; Morin, Le, AI-II, AIII, AIV (Magd VI) ;; Tourasse, La.

Azilien - Peyrat, Le, 2nv Riera, La, 27, 29 (Asturien). 
